\title{
The three dimensional Ising spin glass in an external magnetic field: the role of the silent majority
}

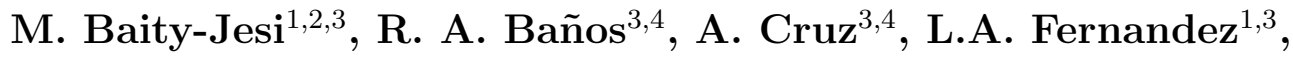 \\ J.M. Gil-Narvion ${ }^{3}$, A. Gordillo-Guerrero ${ }^{3,5}$, D. Iñiguez ${ }^{3,6}$, A. \\ Maiorano $^{2,3}$, F. Mantovani ${ }^{7}$, E. Marinari ${ }^{8}$, V. Martin-Mayor ${ }^{1,3}$, \\ J. Monforte-Garcia ${ }^{3,4}$, A. Muñoz Sudupe ${ }^{1}$, D. Navarro ${ }^{9}$, G. \\ Parisi $^{8}$, S. Perez-Gaviro ${ }^{3}$, M. Pivanti ${ }^{7}$, F. Ricci-Tersenghi ${ }^{8}$, J.J. \\ Ruiz-Lorenzo $^{10,3}$, S.F. Schifano ${ }^{11}$, B. Seoane ${ }^{2,3}$, A. Tarancon ${ }^{3,4}$, \\ R. Tripiccione ${ }^{7}$ and D. Yllanes ${ }^{2,3}$ \\ ${ }^{1}$ Departamento de Física Teórica I, Universidad Complutense, 28040 Madrid, Spain. \\ 2 Dipartimento di Fisica, La Sapienza Università di Roma, 00185 Roma, Italy. \\ ${ }^{3}$ Instituto de Biocomputación y Física de Sistemas Complejos (BIFI), 50009 \\ Zaragoza, Spain. \\ ${ }^{4}$ Departamento de Física Teórica, Universidad de Zaragoza, 50009 Zaragoza, Spain. \\ ${ }^{5}$ D. de Ingeniería Eléctrica, Electrónica y Automática, U. de Extremadura, 10071, \\ Cáceres, Spain. \\ ${ }^{6}$ Fundación ARAID, Diputación General de Aragón, Zaragoza, Spain. \\ ${ }^{7}$ Barcelona Supercomputing Center, 08034 Barcelona, Spain. \\ ${ }^{8}$ Dipartimento di Fisica, IPCF-CNR, UOS Roma Kerberos and INFN, La Sapienza \\ Università di Roma, 00185 Roma, Italy. \\ ${ }^{9}$ D. de Ingeniería, Electrónica y Comunicaciones and I3A, U. de Zaragoza, 50018 \\ Zaragoza, Spain. \\ 10 Departamento de Física, Universidad de Extremadura, 06071 Badajoz, Spain. \\ ${ }^{11}$ Dipartimento di Matematica e Informatica, Università di Ferrara and INFN, \\ Ferrara, Italy.
}

\begin{abstract}
We perform equilibrium parallel-tempering simulations of the 3D Ising Edwards-Anderson spin glass in a field, using the Janus computer. A traditional analysis shows no signs of a phase transition. Yet, we encounter dramatic fluctuations in the behaviour of the model: Averages over all the data only describe the behaviour of a small fraction of it. Therefore we develop a new approach to study the equilibrium behaviour of the system, by classifying the measurements as a function of a conditioning variate. We propose a finite-size scaling analysis based on the probability distribution function of the conditioning variate, which may accelerate the convergence to the thermodynamic limit. In this way, we find a non-trivial spectrum of behaviours, where a part of the measurements behaves as the average, while the majority of them shows signs of scale invariance. As a result, we can estimate the temperature interval where the phase transition in a field ought to lie, if it exists. Although this would-be critical regime is unreachable with present resources, the numerical challenge is finally well posed.
\end{abstract}




\section{Contents}

1 Introduction 4

2 Extended introduction: giant fluctuations and the silent minority 5

2.1 Foreword .................... . . 5

2.2 No signs of a phase transition with common tools . . . . . . . . . . . 6 6

2.3 Signs of a hidden behaviour . . . . . . . . . . . . . 6 6

2.4 Giant fluctuations . . . . . . . . . . . . . . . . . 6

3 Model and simulations 8

3.1 The model . . . . . . . . . . . . . . . . . 8

3.2 The simulations . . . . . . . . . . . . . . . . . . . . 9 9

4 Observables $\quad 10$

5 Finite-size scaling $\quad 12$

6 Conditional expectation values and variances $\mathbf{1 3}$

6.1 The conditioning variate . . . . . . . . . . . . . . . . . . 13

6.2 Measurements against samples . . . . . . . . . . . . . . . 14

6.3 The selection of the conditioning variate . . . . . . . . . . . . 15

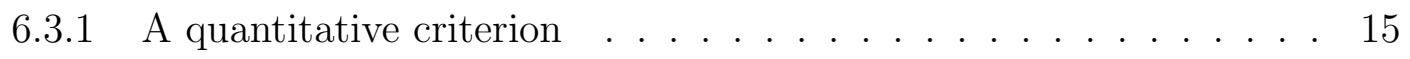

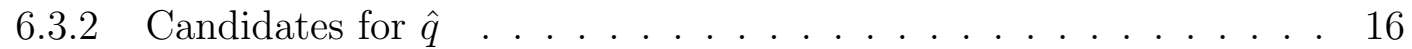

7 Quantiles and a modified finite-size scaling ansatz 18

7.1 The $P\left(q_{\text {med }}\right) \ldots \ldots \ldots \ldots \ldots$. . . . . . . . . . . . . . . . . . . . . . .

8 Results $\quad[19$

9 This is not an echo of the $h=0$ transition 21

9.1 An escaping transition . . . . . . . . . . . . . . . . . . . . . . . . . . .

9.2 Scaling at $T=T_{\mathrm{c}}(h=0) \ldots \ldots \ldots \ldots \ldots$. . . . . . . . . . . . . . .

10 Conclusions

Appendix A. Quantiles for different fields

Appendix B. 4-replica correlators $\quad[29$

Appendix C. Technical details on the creation of quantiles 33

Appendix D. A caveat for the quantile description 34

Appendix E. Finding a privileged $q$ 
CONTENTS

Appendix F. Quantiles with 2-replica correlators

Appendix G. High-temperature extrapolations

References

40 


\section{Introduction}

Spin glasses are disordered magnetic alloys [1]. The most popular model for these alloys is the Edwards-Anderson model [2, 3], widely regarded as one of the simplest instances of a complex system [4]. The corresponding phase diagram is tridimensional: Besides the temperature and the externally applied magnetic field, also the space dimensionality plays a crucial role [4].

Above the upper critical space dimension, $D_{\mathrm{u}}=6$, mean-field theory becomes quantitatively accurate [4, 5, 6]. As for most magnetic systems, time-reversal symmetry is spontaneously broken below a critical temperature $T_{\mathrm{c}}$. Yet, the behaviour of meanfield spin glasses in an external field $h$ is most peculiar. $\$$ Although the external field explicitly breaks time-reversal symmetry at any temperature, de Almeida and Thouless have shown that a phase transition occurs also for $h>0$ at the so-called dAT line, $T_{\mathrm{c}}(h)$ [5]. The symmetry that is spontaneously broken at $T_{\mathrm{c}}(h)$ is the abstract replica symmetry, which encodes a complex free-energy landscape [4, 7, , 8, 9].

It is still unclear how much of the above picture (usually known as Replica Symmetry Breaking or RSB picture) is realised in our three-dimensional world. Some believe it should still apply without dramatic modifications [6, 10]. Yet, a dissenting school of thought, the so-called droplet picture, predicts no phase transition in a field as soon as one goes below six spatial dimensions [11, 12, 13, 14]. Some recent developments of this debate are in [10, 15, 16].

A rather obvious way out would be the experimental study of spin glasses in a

field. Unfortunately, opposing indications have been gleaned over the existence of a phase transition [17, 18, 19, 20].

The Renormalisation Group approach to this problem also provides conflicting results. No fixed points were found by enforcing that the number of replicas of the replicated field theory be zero [21]. However, fixed points were found relaxing this condition and using the most general Hamiltonian [22]. Reasoning along this line, in [23] (see also [10]) the de Almeida-Thouless line was computed for $D$ slightly below $D_{\mathrm{u}}=6$ (the upper critical dimension remains 6 when an external magnetic field is applied).

Equilibrium numerical simulations offer an alternative approach, which has already been effective in establishing that a phase transition does occur at zero field in the $D=3$ Edwards-Anderson model [24, 25] (in agreement with experiments [26]). The same strategy has been followed for $h>0$, with negative results [27, 28]. Yet, this cannot be the whole story: Recent work in $D=4$, hence below $D_{\mathrm{u}}$, using a non-standard finitesize scaling method has found clear evidence for a dAT line [29]. Furthermore, one may try to interpolate between $D=3$ and $D=4$ by tuning long-range interactions in $D=1$ chains [30, 31]. This approach suggests that a dAT might be present in $D=4$, but not in $D=3$ 32] (yet, see the criticism in [33]).

The problem being still open, in [34] we undertook a dynamical study of the $\ddagger$ The definition of $h$ is given in section 3 , (1). 
3-dimensional Edwards-Anderson spin glass with the Janus dedicated computer, an FPGA-based machine especially designed for Ising spin glass simulations [35, 36, 37, 38, We studied very large lattices $(L=80$ ), in wide time scales (from an equivalent of $\sim 1 \mathrm{ps}$ to $\sim 0.01 \mathrm{~s}$ ), and gathered both equilibrium and non-equilibrium data. We focused on the increase of relaxation times and found a would-be dynamical transition, but at a suspiciously high temperature. A subsequent examination of the correlation length found a growth faster than predicted by the droplet theory, and slower than what RSB would expect. We also examined the problem from a supercooled liquid point of view [39, 40, 41, 42, 43, 44], motivated by [45, 46]. At any rate, the study of the possible critical divergence of the correlation length allowed us to give upper bounds $T^{\mathrm{up}}(h)$ to the possible transition line for the studied fields $\$$ For further reference we recall that $T^{\mathrm{up}}(h=0.1)=0.8$ and $T^{\mathrm{up}}(h=0.2)=T^{\mathrm{up}}(h=0.3)=0.5$.

The impossibility to get concluding evidence in [34], may be due to the fact that we did not reach low enough temperatures (our simulations fell out of equilibrium at temperatures $T$ significantly higher than $T^{\mathrm{up}}(h)$ ). In any case, a study of the equilibrium properties of the model is mandatory if one wants to understand the nature of the thermodynamic phases of the three-dimensional Edwards-Anderson spin glass in a field.

In this paper we report the result of equilibrium simulations performed on Janus, using lattices up to $L=32$. Analogously to what has been already found in meanfield spin glasses on the de Almeida-Thouless line, we find extreme fluctuations in the model's behaviour [47]. We will propose a method to tame these fluctuations, and we will find out that, although the average behaviour does not show any sign of a phase transition, this is not true for the medians of our observables, where we have indications of a possible phase transition at a temperature $T_{\mathrm{c}} \lesssim T^{\mathrm{up}}(h)$.

In section 2 we make an extension of this introduction, giving an intuitive justification of why we deepened our analysis after finding no sign of a phase transition using the standard indicators. We define the model and explain how simulations were done in section 3. In section 4 we define the observables we measured, and we make a brief parenthesis on finite-size scaling in section 5. Sections 6 and 7 are dedicated to the description of the method we adopted to monitor extreme fluctuations. In section 8 we present our results, and in section 9 we show that they are not an echo of the zero-field transition. Finally we give our conclusions in section 10 . Further details are given in the appendices.

\section{Extended introduction: giant fluctuations and the silent minority}

\subsection{Foreword}

Since the bulk of the article will get technical in the description of a method that allows a classification of the data, we will first give a qualitative description of our results in

$\S$ In 34 we studied a bimodal field, while in this paper $h$ is constant. Notwithstanding, we will make comparisons with the bounds $T^{\mathrm{up}}(h)$ by matching $\overline{h^{2}}$ in both models. 
terms of intuitive concepts. In this way, the reader will be more aware of the reasons that lead to the conception of the following analysis.

\subsection{No signs of a phase transition with common tools}

The most common way to locate a phase transition is to identify some observable $R$ that benefits from scale invariance in the presence of a phase transition (for example the correlation length $\xi_{L}$ measured in a lattice of size $L$, divided by $L$ ). This means that if we plot $R$ as a function of the temperature, for many system sizes, all the curves will cross at the critical temperature $T_{\mathrm{c}}$ where the phase transition occurs. For sufficiently large systems, if the curves do not cross, there is no phase transition in the simulated temperature range (see sections 4 and 5 for details). The idea dates back to Nightingale [48] and Binder [49], and has been very successful in the study of disordered systems $[25,28,50,51,52,53,54,51,55,56,57$. 5.

In our case, this type of analysis yields a clear result: there is no evidence of a crossing at the simulated temperatures, magnetic fields and sizes. This is clearly visible from figure 1 where the curves $\xi_{L}(T) / L$ and $R_{12}(T)$ [see (10) and (11) in section 4 ] should have some crossing point if we were in the presence of a phase transition. This is in complete qualitative agreement with earlier works on this model [27, 28].

\subsection{Signs of a hidden behaviour}

Although $\xi_{L}(T) / L$ is smaller the larger the lattice size, the coherence length $\xi_{L}$ grows significantly even for our largest lattice sizes. For example at $h=0.2, T=0.81$ we have $\xi_{16}=6.09(4), \xi_{24}=7.63(9)$ and $\xi_{32}=9.0(2)$. The noticeable size evolution implies that the asymptotic correlation length $\xi_{\infty}$ is large compared with $L=32$.

Also, we can examine the behaviour of the spin-glass order parameter, the overlap $q$, by studying its distribution function $P(q)$. In the absence of a phase transition we would be in the paramagnetic phase, and $P(q)$ should be a delta function of a positive overlap $q_{\mathrm{EA}}$ (so in finite systems it should be Gaussian).

Instead, we can see from figure 2 that its distribution $P(q)$ has a very wide support, with tails that, for small enough magnetic fields, reach even negative values of $q$. This is precisely what was observed in the mean-field version of the model on the de AlmeidaThouless line, and it was attributed to the contribution of few samples [47].

From these arguments it becomes reasonable to think that we may not be simulating large enough lattices to observe the asymptotic nature of the system and that there may be some hidden behaviour that we are not appreciating.

\subsection{Giant fluctuations}

In fact, we find out that the average values we measure are representative of only a small part of the data set. That is, the average of relevant observables (e.g., the 


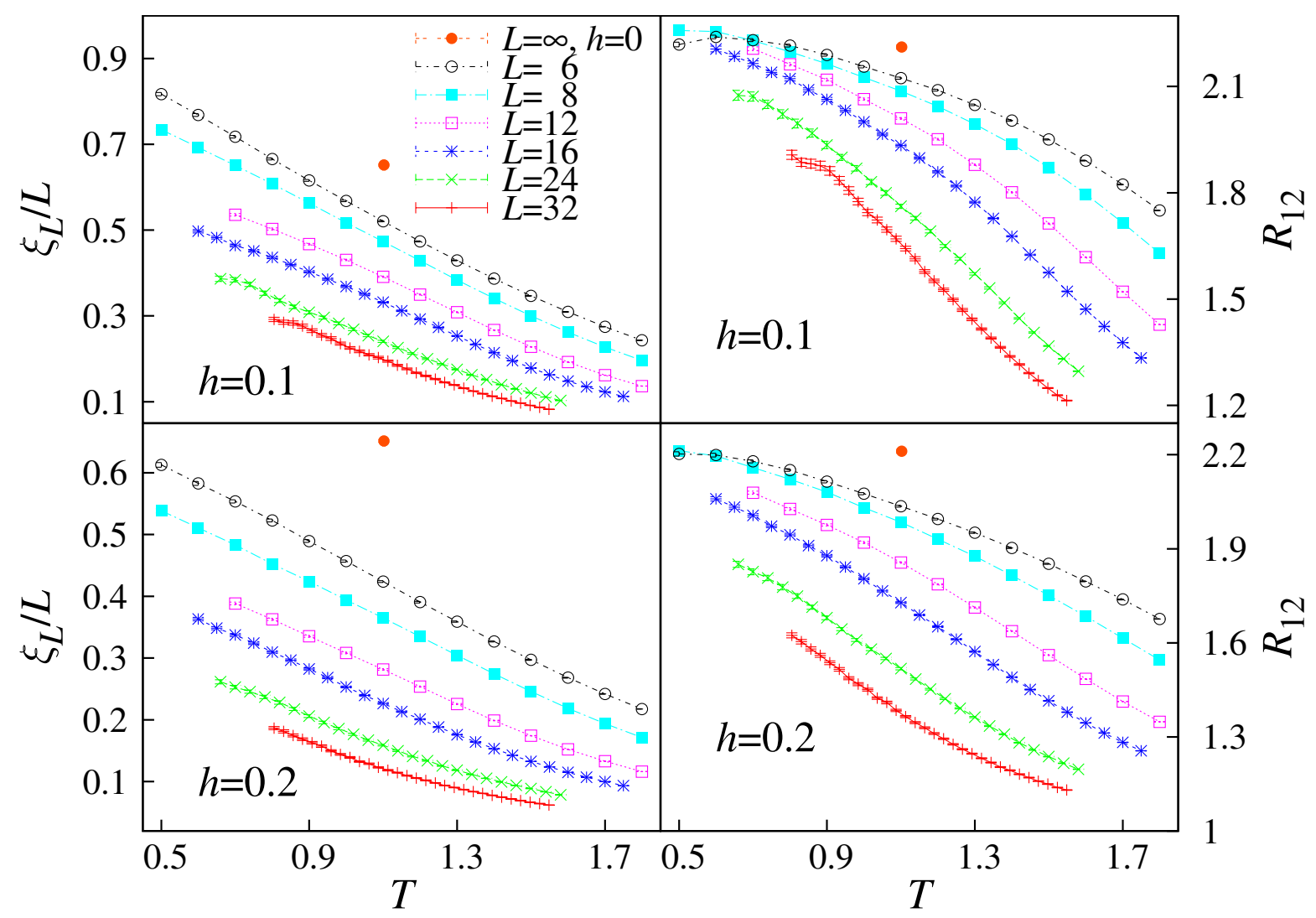

Figure 1. The figures on the left show the standard correlation length $\xi_{L}$ in units of the lattice size $L$ as a function of the temperature $T$, for all our lattice sizes. The magnetic fields are $h=0.1$ (top), and $h=0.2$ (bottom). If the lattices are large enough, in the presence of a second-order phase transition, the curves are expected to cross at a finite temperature $T_{\mathrm{c}}(h)$. The figures on the right show the cumulant $R_{12}$, which in the presence of a magnetic field is a better indicator of a phase transition [29], for the same magnetic fields. At zero field the heights of the crossings (which are universal quantities) are indicated with a point at $T_{\mathrm{c}}=1.1019(29)$. They are $\xi_{L} / L\left(h=0 ; T_{\mathrm{c}}\right)=0.6516(32)$ and $R_{12}\left(h=0 ; T_{\mathrm{c}}\right)=2.211(6)$ [51]. In neither case we observe signs of a crossing at the simulated temperatures, nor can we state that the curves will cross at lower temperature. The reader might remark that the curve for $L=32, h=0.1$ is not as smooth as one would expect from parallel tempering simulations. The reason is twofold. On one side the number of simulated samples is much smaller than for $L<32$, and on the other side temperature chaos, which is stronger the larger the lattice, is probably present 58 .

spatial correlation function) only represents the small number of measurements that are dominating it. The rest of the measurements is not appreciated by using the average.

Clearly, standard finite-size scaling methods are not adequate to these systems, and we need to find a way to take into account all the measurements. Recalling the wide distributions of figure 2, it seems reasonable to sort our measurements according to some conditioning variable $\hat{q}$ related to the overlaps between our replicas (see section 6). This way, we find out that the average values we measure are given by only a small part of the measurements. For example in figure 3 we show the correlation function $C(r)$. 


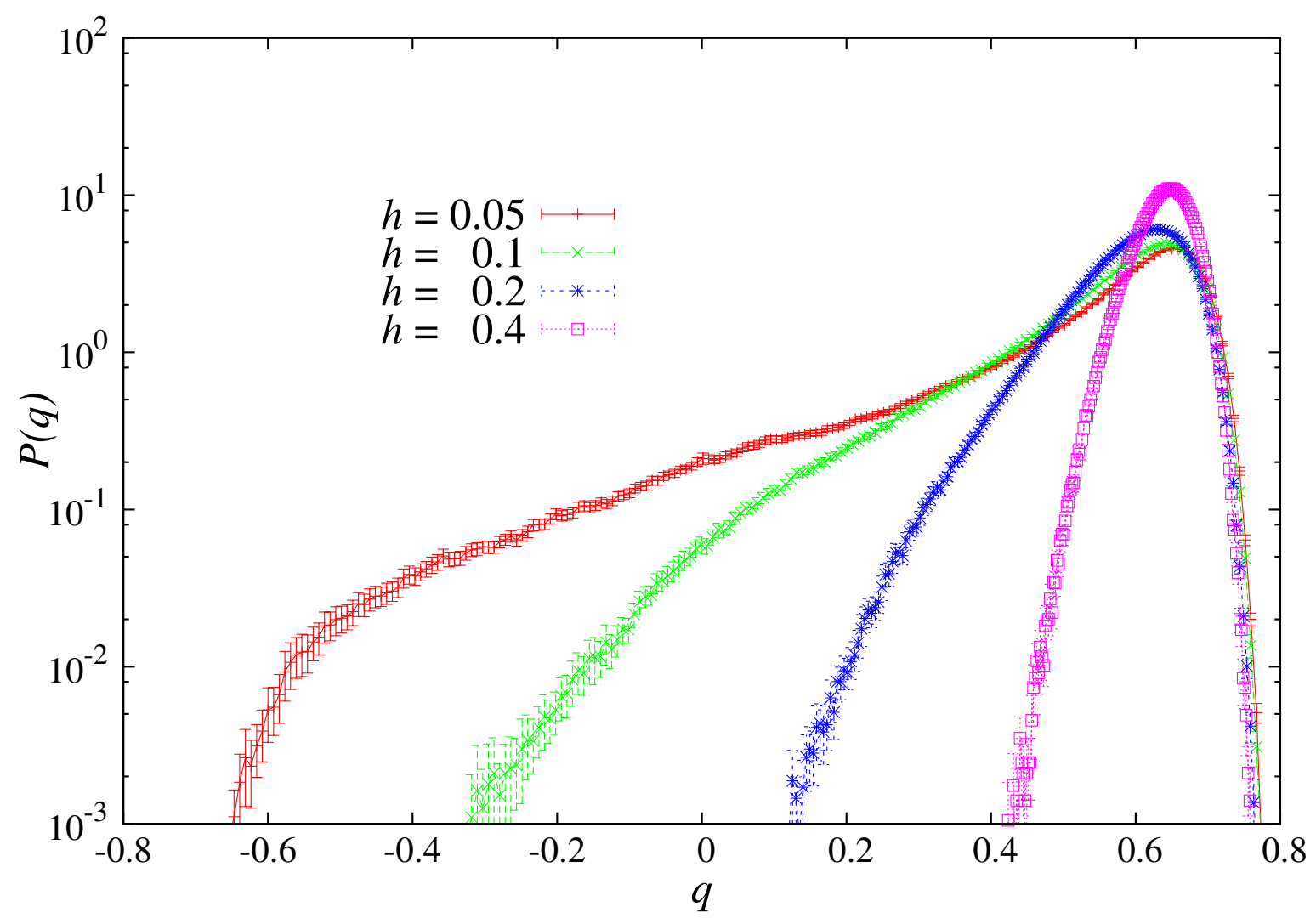

Figure 2. The probability distribution function $P(q)$ of the overlap $q$, for our largest lattices $(L=32)$ at the lowest simulated temperature $(T=0.805128)$, for all our magnetic fields $(h=0.05,0.1,0.2,0.4)$, see table 1 . The order parameter in the Edwards-Anderson model is the overlap $q$, and it is defined in the $[-1,1]$ interval (see section 4). The supports are wide, with exponential tails similar to those in the mean-field model at the dAT transition line [47.

We plot 4 estimators of $C(r)$ : the average (which is the standard quantity studied in almost all, if not all, previous work), the $C(r)$ that corresponds to the median of the $\hat{q}$ distribution, and the measurements with the $10 \%$ highest (lowest) value of $\hat{q}$. We see that the average is very close to the $10 \%$ lowest $\hat{q}$, and very far from the two other curves. So, when we plot the average curve, we are only representing the behaviour of that small set of data.

Therefore, if we want to understand the behaviour of the whole collection of measurements, we have to be able to find some criterion to sort them and analyse them separately.

\section{Model and simulations}

\subsection{The model}

We consider a $3 D$ cubic lattice of size $L$ with periodic boundary conditions. In each of the $V=L^{3}$ vertices of the lattice there is a spin $\sigma_{\boldsymbol{x}}= \pm 1$. The spins interact uniquely 


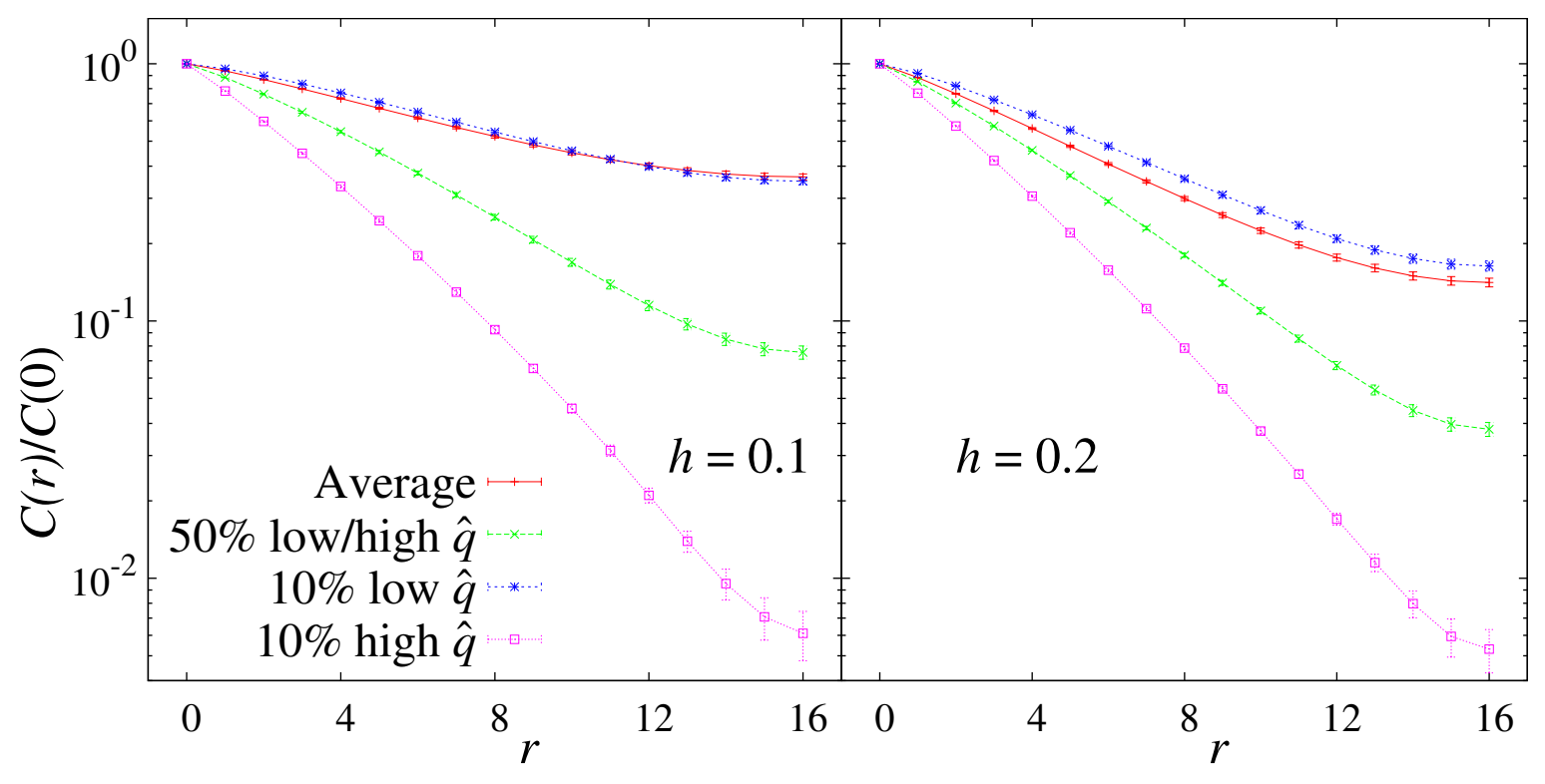

Figure 3. Different instances of the normalised correlation function $C(r)$ (9) for $L=32, T=0.805128$. The field is $h=0.1$ on the left, and $h=0.2$ in the right plot. We sort the measurements with the help of a conditioning variate $\hat{q}$ as described in section 6. In this case $\hat{q}$ is the median overlap $q_{\text {med }}$. We show small sets of measurements. Namely, the ones with the $10 \%$ lowest (top curve) and highest (bottom curve) $\hat{q}$ and those whose $\hat{q}$ corresponds to the median of the distribution of $\hat{q}(50 \%$ lowest/highest $\hat{q})$. This sorting reveals extreme differences in the fauna of measurements. The average and median of the correlation functions are very different. The average is very similar to the $10 \%$ lowest ranked measures, i.e., it is only representative of a very small part of the data. We normalise $C(r)$ by dividing by $C(0)$ because we measure point-to-plane correlation functions (9). The correlation functions have zero slope at $r=L / 2$ due to the periodic boundary conditions.

with their nearest neighbours and with an external magnetic field $h$. The Hamiltonian is

$$
\mathcal{H}=-\sum_{\langle\boldsymbol{x}, \boldsymbol{y}\rangle} J_{x y} \sigma_{x} \sigma_{\boldsymbol{y}}-h \sum_{\boldsymbol{x}} \sigma_{\boldsymbol{x}}
$$

where $\langle\boldsymbol{x}, \boldsymbol{y}\rangle$ means the sums are only over the nearest neighbours, while the couplings $J_{x y}$, which are constant during each simulation, take the values \pm 1 with equal probability (quenched disorder). A given instance of the bonds $J_{x y}$ and of the intensity of the magnetic field $h$ define a sample. We will consider real replicas of each sample, i.e., systems with identical couplings $J_{x y}$ and field $h$, but independent evolutions (for a recent discussion see [36] and [59]). In this work we will use 4 replicas per sample.

\subsection{The simulations}

For all our simulations we made use of parallel tempering (PT) [60, 61]. The whole procedure was very similar to the one in [29].

The smaller lattices $(L=6,8,12)$ were simulated with multispin coding (C code 
with words of 128 bits, by means of streaming extensions) [29, 62] on the Memento CPU cluster at BIFI. The larger samples $(L=16,24,32)$ were simulated on the Janus dedicated computer [38].

An Elementary Monte Carlo Step (EMCS) consisted in 1 PT exchange every 10 Metropolis steps for the multispin-coding samples, and 1 PT every 10 heatbath for the samples simulated on Janus. table 1 shows the relevant parameters of the simulations. The temperatures were equally spaced between $T_{\min }$ and $T_{\max }$. The intensities of the external magnetic field we chose are $h=0.05,0.1,0.2$ and 0.4 .

To check whether the samples were thermalised we measured the exponential selfcorrelation time of the PT random walk in temperatures $\tau$ [29, 36, 63, 64]. We required the simulations to last at least $14 \tau$. To do so without consuming computing time on already thermalised lattices, we assigned a minimum number of EMCS, $N_{\text {MCS }}^{\min }$, for all the samples, and extended by a factor $f>1$ only the ones that did not meet the imposed thermalisation criterion. In table 1 we report $N_{\mathrm{EMCS}}^{\min }$, the maximum extension factor $f_{\max }$ of the simulations, and minimum number $N_{\tau}^{\min }$ of EMCS in units of $\tau$.

Equilibrium measures were taken offline over the second half of each simulation. Independently of how much the simulations were extended, we saved 16 equally timespaced configurations and performed measures on them. We were measuring 4-replica observables. Therefore, for each sample it was possible to choose quadruplets of configurations, each from a different replica, in $16^{4}$ ways. Out of the $16^{4}$ possibilities, we chose randomly $N_{\mathrm{t}}=1000$ combinations. In other words, each sample participated in the statistics with $N_{\mathrm{t}}=1000$ measurements.

The errors were estimated with the jackknife method.

\section{Observables}

For each sample we simulated 4 different replicas, in order to be able to compute connected correlation functions that go to zero at infinite distance. We will label replicas by using superscripts, this means that the generic quantity $\mathcal{O}^{(a)}$ belongs to the $a^{\text {th }}$ replica of a given sample.

We will denote with over-lines $\overline{(\ldots)}$ the averages over the samples, and with brackets $\langle\ldots\rangle$ the thermal averages. To make notation less heavy we will use $E(\ldots)=\overline{\langle\ldots\rangle}$ to denote an average that is taken first over the thermal fluctuations and then over the samples.

We define the local overlap as

$$
q_{\boldsymbol{x}}^{(\mathrm{ab})}=\sigma_{\boldsymbol{x}}^{(a)} \sigma_{\boldsymbol{x}}^{(b)},
$$

while the total overlap is

$$
q^{(a b)}=\frac{1}{V} \sum_{\boldsymbol{x}} q_{\boldsymbol{x}}^{(a b)} .
$$


Table 1. Parameters of the simulations. We report the magnetic field $h$, the lattice linear size $L$, the number of simulated samples $N_{\text {samples }}$, and the basic length of a simulation in Elementary Monte Carlo Steps $N_{\text {EMCS }}^{\min }$. In each simulation we measured the exponential correlation time $\tau$ of the PT random walk in temperatures. When $\tau$ was too large to meet our thermalisation requirements, we extended the length of each simulation by an extension factor $f$. We denote with $f_{\max }$ the greatest extension factor. We also give the minimum length of a simulation $N_{\tau}^{\min }$ in units of $\tau$. In all cases we imposed $N_{\tau}^{\min }>14$. Finally, we give the number of temperatures $N_{T}$ we used for the PT, and the minimum and maximum temperatures $T_{\min }$ and $T_{\max }$.

\begin{tabular}{|c|c|c|c|c|c|c|c|c|}
\hline$h$ & $L$ & $N_{\text {samples }}$ & $N_{\mathrm{EMCS}}^{\min }$ & $f_{\max }$ & $N_{\tau}^{\min }$ & $N_{\mathrm{T}}$ & $T_{\min }$ & $T_{\max }$ \\
\hline 0.05 & 6 & 25600 & $1.6 \times 10^{6}$ & 1 & 40.0 & 14 & 0.5 & 1.8 \\
\hline 0.05 & 8 & 25600 & $3.2 \times 10^{6}$ & 16 & 40.0 & 14 & 0.5 & 1.8 \\
\hline 0.05 & 12 & 25600 & $3.2 \times 10^{6}$ & 16 & 15.6 & 12 & 0.7 & 1.8 \\
\hline 0.05 & 16 & 12800 & $1.28 \times 10^{7}$ & 128 & 20.1 & 24 & 0.6 & 1.75 \\
\hline 0.05 & 24 & 6400 & $1.28 \times 10^{7}$ & 110 & 16.0 & 20 & 0.78 & 1.54 \\
\hline 0.05 & 32 & 2400 & $6.4 \times 10^{7}$ & 256 & 14.3 & 30 & 0.805128 & 1.54872 \\
\hline 0.1 & 6 & 25600 & $1.6 \times 10^{6}$ & 4 & 40.0 & 14 & 0.5 & 1.8 \\
\hline 0.1 & 8 & 25600 & $3.2 \times 10^{6}$ & 16 & 40.0 & 14 & 0.5 & 1.8 \\
\hline 0.1 & 12 & 25600 & $3.2 \times 10^{6}$ & 16 & 14.4 & 12 & 0.7 & 1.8 \\
\hline 0.1 & 16 & 12800 & $1.28 \times 10^{7}$ & 256 & 27.9 & 24 & 0.6 & 1.75 \\
\hline 0.1 & 24 & 3200 & $1.28 \times 10^{7}$ & 4097 & 14.3 & 24 & 0.66 & 1.58 \\
\hline 0.1 & 32 & 1600 & $6.4 \times 10^{7}$ & 533 & 14.4 & 30 & 0.805128 & 1.54872 \\
\hline 0.2 & 6 & 25600 & $1.6 \times 10^{6}$ & 1 & 40.0 & 14 & 0.5 & 1.8 \\
\hline 0.2 & 8 & 25600 & $3.2 \times 10^{6}$ & 16 & 40.0 & 14 & 0.5 & 1.8 \\
\hline 0.2 & 12 & 25600 & $3.2 \times 10^{6}$ & 64 & 25.4 & 12 & 0.7 & 1.8 \\
\hline 0.2 & 16 & 12800 & $1.28 \times 10^{7}$ & 256 & 18.4 & 24 & 0.6 & 1.75 \\
\hline 0.2 & 24 & 3200 & $1.28 \times 10^{7}$ & 512 & 16.1 & 24 & 0.66 & 1.58 \\
\hline 0.2 & 32 & 1600 & $1.6 \times 10^{7}$ & 513 & 16.0 & 30 & 0.805128 & 1.54872 \\
\hline 0.4 & 6 & 25600 & $1.6 \times 10^{6}$ & 1 & 40.0 & 14 & 0.5 & 1.8 \\
\hline 0.4 & 8 & 25600 & $3.2 \times 10^{6}$ & 4 & 30.7 & 14 & 0.5 & 1.8 \\
\hline 0.4 & 12 & 25600 & $3.2 \times 10^{6}$ & 16 & 14.1 & 12 & 0.7 & 1.8 \\
\hline 0.4 & 16 & 3200 & $1.28 \times 10^{7}$ & 32 & 20.1 & 24 & 0.6 & 1.75 \\
\hline 0.4 & 24 & 800 & $1.28 \times 10^{7}$ & 29 & 16.1 & 24 & 0.66 & 1.58 \\
\hline 0.4 & 32 & 800 & $3.2 \times 10^{6}$ & 16 & 16.4 & 30 & 0.805128 & 1.54872 \\
\hline
\end{tabular}

We show in Appendix B that the most informative connected correlator we can construct with 4 replicas is the replicon propagator [5, 65.

$$
G_{\mathrm{R}}(\boldsymbol{r})=\frac{1}{V} \sum_{\boldsymbol{x}} \overline{\left(\left\langle\sigma_{\boldsymbol{x}} \sigma_{\boldsymbol{x}+\boldsymbol{r}}\right\rangle-\left\langle\sigma_{\boldsymbol{x}}\right\rangle\left\langle\sigma_{\boldsymbol{x}+\boldsymbol{r}}\right\rangle\right)^{2}} .
$$

To compute $G_{\mathrm{R}}$ we calculate the 4-replica field

$$
\Phi_{x}^{(a b ; c d)}=\frac{1}{2}\left(\sigma_{x}^{(a)}-\sigma_{x}^{(b)}\right)\left(\sigma_{x}^{(c)}-\sigma_{x}^{(d)}\right),
$$


where the indices $a, b, c, d$ indicate strictly different replicas. Notice that

$$
\left\langle\Phi_{\boldsymbol{x}}^{(a b ; c d)} \Phi_{\boldsymbol{y}}^{(a b ; c d)}\right\rangle=\left(\left\langle\sigma_{\boldsymbol{x}} \sigma_{\boldsymbol{x}+\boldsymbol{r}}\right\rangle-\left\langle\sigma_{\boldsymbol{x}}\right\rangle\left\langle\sigma_{\boldsymbol{x}+\boldsymbol{r}}\right\rangle\right)^{2}
$$

so we obtain $G_{\mathrm{R}}$ by taking also the average over the samples

$$
E\left(\Phi_{\boldsymbol{x}}^{(a b ; c d)} \Phi_{\boldsymbol{y}}^{(a b ; c d)}\right)=G_{\mathrm{R}}(\boldsymbol{x}-\boldsymbol{y}) .
$$

Here, and everywhere there is more than one possible permutation of the replica indices, we average over all of them to gain statistics.

Correlations in the Fourier space are defined analogously, by Fourier-transforming $\Phi_{x}^{(a b ; c d)}$, so the wave-vector dependent replicon susceptibility is expressed as

$$
\chi_{\mathrm{R}}(\boldsymbol{k})=\frac{1}{V} E\left(\left|\hat{\Phi}_{\boldsymbol{k}}^{(a b ; c d)}\right|^{2}\right) \quad, \quad \hat{\Phi}_{\boldsymbol{k}}^{(a b ; c d)}=\sum_{\boldsymbol{x}}^{V} \mathrm{e}^{\mathrm{i} \boldsymbol{k} \cdot \boldsymbol{x}} \Phi_{\boldsymbol{x}}^{(a b ; c d)} .
$$

When we omit $\boldsymbol{k}$, we refer to the susceptibility $\chi=\chi_{\mathrm{R}}(\boldsymbol{O})$.

We compute point-to-plane correlation functions

$$
C(r)=\frac{1}{L} \sum_{n=0}^{L-1} \mathrm{e}^{-\mathrm{i} r 2 \pi n / L} \chi_{\mathrm{R}}\left(\frac{2 \pi n}{L}, 0,0\right) \equiv \sum_{y, z} G_{\mathrm{R}}(x=r, y, z) .
$$

The previous relation is equivalent if we align the wave vector along any of the three coordinate axes, so we average over these three choices.

With the defined observables we were able to calculate the second-moment correlation length

$$
\xi_{L}=\frac{1}{2 \sin \left(k_{\min } / 2\right)} \sqrt{\frac{\chi_{\mathrm{R}}(\mathbf{0})}{\chi_{\mathrm{R}}(2 \pi / L, 0,0)}-1},
$$

and the dimensionless quantity

$$
R_{12}=\frac{\chi_{\mathrm{R}}(2 \pi / L, 0,0)}{\chi_{\mathrm{R}}(2 \pi / L, \pm 2 \pi / L, 0)}
$$

where all the quantities were averaged over all the possible permutations of the components of the wave vectors.

The cumulant $R_{12}$ (recall figure 1) was used in [29] to estimate the critical temperature bypassing pathologies on $\chi(\mathbf{0})$ due to the fact that the overlap is non-zero in the paramagnetic phase (recall figure 2).

\section{Finite-size scaling}

Our finite-size scaling analysis follows phenomenological renormalisation, which is very effective when one looks for a phase transition with a diverging correlation length [66, 48, 67]. In fact, under the accepted assumption that there is only one relevant length scale in the system, close to the critical temperature $T_{\mathrm{c}}$ a generic observable $\mathcal{O}$ scales like

$$
\langle\mathcal{O}(L, T)\rangle=L^{x_{\mathcal{O}} / \nu}\left[\mathcal{F}_{\mathcal{O}}\left(L^{1 / \nu}\left(T-T_{\mathrm{c}}\right)\right)+O\left(L^{-\omega}\right)\right]
$$


where $\omega>0$ represents the leading irrelevant exponent.

This implies that at the critical point the quantities $\xi_{L}(T, L, h) / L$ and $R_{12}(T, L, h)$ are scale invariant to the dominant order:

$$
\begin{aligned}
& \frac{\xi_{L}}{L}(L, T)=\mathcal{F}_{\xi}\left(L^{1 / \nu}\left(T-T_{\mathrm{c}}\right)\right)+\ldots, \\
& R_{12}(L, T)=\mathcal{F}_{R}\left(L^{1 / \nu}\left(T-T_{\mathrm{c}}\right)\right)+\ldots,
\end{aligned}
$$

where the dots indicate subleading corrections to scaling. So, for large enough systems the critical point at given $h$ would be revealed by the crossing points of these curves for different sizes. As figure 1 shows, such a crossing is not present in our data.

With the use of the hyperscaling relations we can also predict the scaling of the susceptibility close to the critical point:

$$
\chi_{\mathrm{R}}(L, T)=L^{2-\eta} \mathcal{F}_{\chi}\left(L^{1 / \nu}\left(T-T_{\mathrm{c}}\right)\right)+\ldots .
$$

\section{Conditional expectation values and variances}

\subsection{The conditioning variate}

As we pointed out in section 2, the behaviour of the system is dominated by a very small number of measurements.

This means that the average over all the measurements of an observable does not describe the typical behaviour of the system. Furthermore, the behaviour of the measurements that contribute less to the full averages is qualitatively different from the one of those who give the main contribution (see figure 3 and later on section 8).

We want to classify our measurements in a convenient way, in order to be able to separate different behaviours, and analyse them separately. To this goal, we replace normal expectation values $E(\mathcal{O})$ of a generic observable $\mathcal{O}$, with the expectation value $E(\mathcal{O} \mid \hat{q})$ conditioned to another random variable $\hat{q}$. Perhaps for lack of imagination $\hat{q}$ will be named conditioning variate. For each instance of $\mathcal{O}$ we monitor also the value of $\hat{q}$, and we use it to label $\mathcal{O}$. Hopefully, there will be some correlation.

The conditional expectation value is defined as the average of $\mathcal{O}$, restricted to the measurements $i$ (out of the $\mathcal{N}_{\mathrm{m}}=N_{\mathrm{t}} N_{\text {samples }}$ total measurements) that simultaneously yield $\mathcal{O}_{i}$ and $\hat{q}_{i}$ [so we are actually talking about couples of simultaneous measurements $\left.\left(\mathcal{O}_{i}, \hat{q}_{i}\right)\right]$ in a small interval around $\hat{q}=c$,

$$
E(\mathcal{O} \mid \hat{q}=c)=\frac{E\left[\mathcal{O}_{i} \mathcal{X}_{\hat{q}=c}\left(\hat{q}_{i}\right)\right]}{E\left[\mathcal{X}_{\hat{q}=c}\left(\hat{q}_{i}\right)\right]} .
$$

Where we have used the characteristic function

$$
\mathcal{X}_{c}\left(\hat{q}_{i}\right)= \begin{cases}1, & \text { if }\left|c-\hat{q}_{i}\right|<\epsilon \sim \frac{1}{\sqrt{V}} \\ 0, & \text { otherwise. }\end{cases}
$$

In Appendix C we give technical details on the choice of $\epsilon$. To make notation lighter, in the rest of the paper we will replace $E(\mathcal{O} \mid \hat{q}=c)$ with $E(\mathcal{O} \mid \hat{q})$. 
The traditional expectation value $E(\mathcal{O})$ can be recovered by integrating over all the possible values of the conditioning variate $\hat{q}$ :

$$
E(\mathcal{O})=\int \mathrm{d} \hat{q} E(\mathcal{O} \mid \hat{q}) P(\hat{q}), P(\hat{q})=E\left[\mathcal{X}_{\hat{q}}\right],
$$

where $P(\hat{q})$ is the probability distribution function of the conditioning variate.

We remark that the concept of conditioning variate is fairly similar to the one of control-variate. Yet, the latter was formalised slightly differently, and with the objective of enhancing the precision of the measures [68]. In [36, 69] a procedure very similar to the present one was followed, but the aim was constructing clustering correlation functions, while in our case the conditioning variate is used to analyse separately different behaviours outcoming from the same global data set, so that a sensible finite-size scaling becomes possible.

\subsection{Measurements against samples}

The reader may argue that a sample-to-sample distinction of the different behaviours is more natural than a measurement-dependent one (although intuition leads to assume

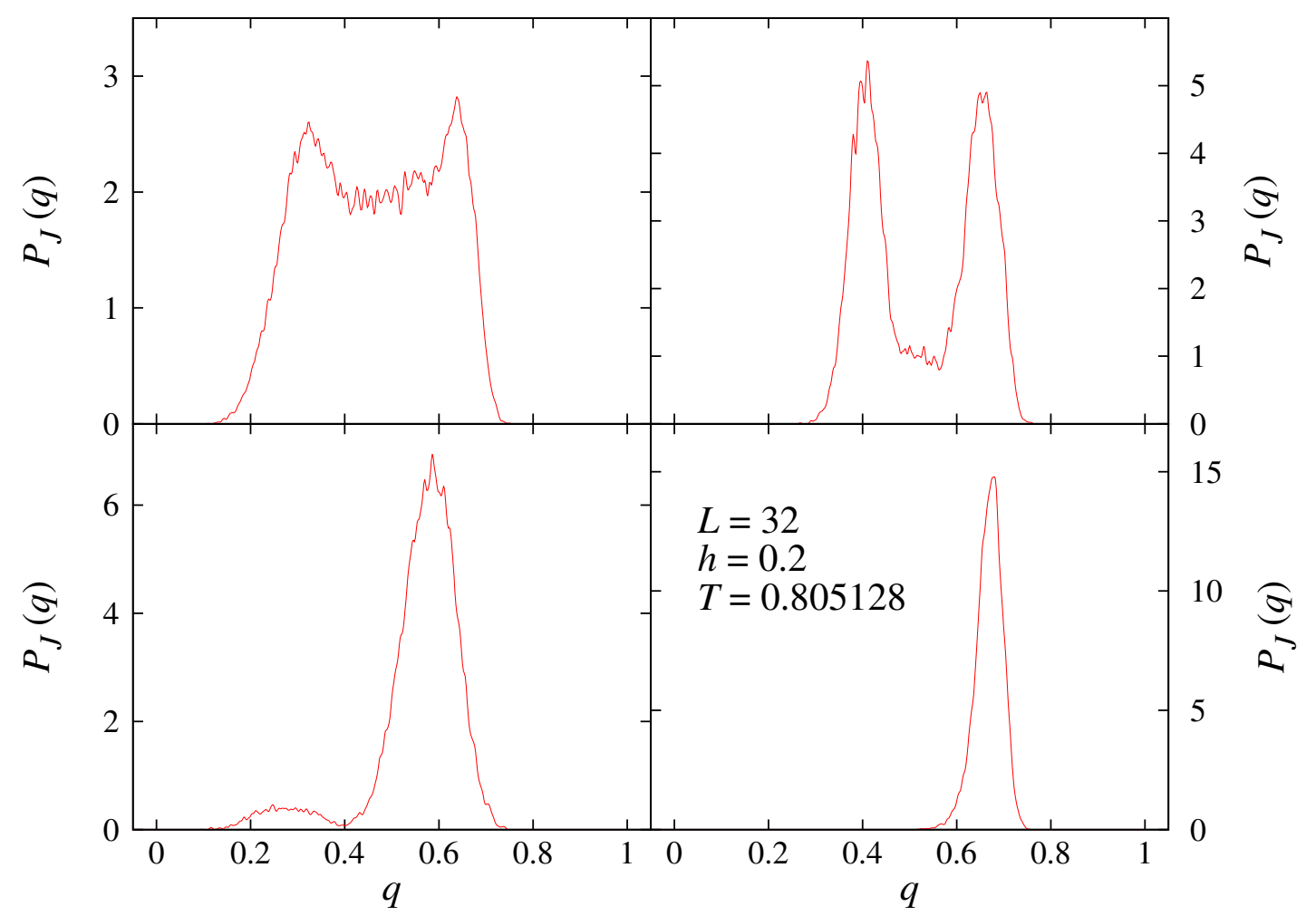

Figure 4. Sample-dependent probability distribution functions $P_{J}(q)$, for four different samples, each representing a different type of $P_{J}(q)$ we encountered. As well as the averaged $P(q)$, also the sample-dependent density function can be wide and with a structure. The plotted data comes from samples with $L=32, h=0.2$ and $T=0.805128$. 
that the two are related). This was indeed our first approach to the problem (it was, in fact, proposed in Ref. [47]). However, we found that the approach described in the previous section is preferable, both for practical and conceptual reasons.

On the practical side, a sample-to-sample separation implies that from each sample we get only one data point: For any observable, we limit ourselves to its thermal average. In this case we would need a limitless amount of samples to be able to construct a reasonable $P(\hat{q})$. Moreover, the simulations should last a huge number of autocorrelation times $\tau$ if we want to have small enough errors on the thermal averages of each sample. Otherwise, we would introduce a large bias that is not reduced when increasing the number of samples.

On the conceptual side, representing each sample merely with a single number (namely the thermal expectation value), is a severe oversimplification. As we show in Fig. 4, even though we are in the paramagnetic phase, the behaviour within each sample is far from trivial. For a non-negligible fraction of the samples, the overlap distribution is wide, often with a multi-peak structure. The barriers among peaks can be deep, hence suggesting extremely slow dynamics (which is indeed the case for physical dinamics, Ref. [34, or for the parallel tempering dynamics, section 3].

In summary, we find that using instantaneous measurements to classify the available information is the best solution.

\subsection{The selection of the conditioning variate}

6.3.1. A quantitative criterion A convenient conditioning variate is the one that mostly discerns the different behaviours of the model. We can get a quantitative criterion for the selection of a good $\hat{q}$ by examining the following relation for any conditional variance:

$$
\operatorname{var}(\mathcal{O})=c_{1}+c_{2}
$$

where we defined

$$
\begin{aligned}
& c_{1} \equiv \int_{-1}^{1} \mathrm{~d} \hat{q} P(\hat{q}) \operatorname{var}(\mathcal{O} \mid \hat{q}), \quad \operatorname{var}(\mathcal{O} \mid \hat{q})=E\left([\mathcal{O}-E(\mathcal{O} \mid \hat{q})]^{2} \mid \hat{q}\right), \\
& c_{2} \equiv \int_{-1}^{1} \mathrm{~d} \hat{q} P(\hat{q})[E(\mathcal{O})-E(\mathcal{O} \mid \hat{q})]^{2} .
\end{aligned}
$$

Both $c_{1}$ and $c_{2}$ are positive, and their sum is fixed.

Let us explain intuitively why a useful conditioning variate has $c_{2} \gg c_{1}$.

If $c_{1}=0$ the fluctuations of $\mathcal{O}$ would be explained solely by the fluctuations of $\hat{q}$. In this case $c_{2}$ is large and assume its largest possible value, meaning that different values of $\mathcal{O}$ are mostly spread apart by $\hat{q}$.

On the other side, $c_{2}=0$ implies $E(\mathcal{O})=E(\mathcal{O} \mid \hat{q})$ and signals an insensitive conditioning variate, with null correlation between $\mathcal{O}$ and $\hat{q}$.

Equations (19) and (20) can thus be used to quantify the quality of the conditioning variate $\hat{q}$ : We look for the highest quotient $c_{2} / c_{1}$. 
Table 2. Criterion for the choice of the conditioning variate $\hat{q}$ for $h=0.1, L=32$, $T=0.805128$, by looking at the indicators $c_{1}$ and $c_{2}$ relatively to $\chi_{\mathrm{R}}(\mathbf{0})$. We want the $\hat{q}$ to split as much as possible the different measured susceptibilities. This is obtained, see $(20)$, when the ratio $c_{2} / c_{1}$ is maximised. From the data we see that this occurs with $\hat{q}=q_{\text {med }}$.

\begin{tabular}{cccc}
$\hat{q}$ & $c_{1}$ & $c_{2}$ & $c_{2} / c_{1}$ \\
\hline \hline$q_{\min }$ & $399000 \pm 37000$ & $121000 \pm 15000$ & $0.30(6)$ \\
$q_{\max }$ & $514000 \pm 51000$ & $6230 \pm 690$ & $0.012(3)$ \\
$q_{\text {med }}$ & $162000 \pm 10000$ & $358000 \pm 45000$ & $2.2(4)$ \\
$q_{\text {av }}$ & $328000 \pm 26000$ & $192000 \pm 28000$ & $0.6(1)$
\end{tabular}

6.3.2. Candidates for $\hat{q}$ To select an appropriate conditioning variate we need to chose $\mathcal{O}$ and propose some test definitions for $\hat{q}$. The functions of the observables that one could use as a conditioning variate are infinite, but physical intuition lead us to try with simple functions of the overlap. On the other side, a natural choice of $\mathcal{O}$ is the estimator of the replicon susceptibility [see (8)]. This means that

$$
\mathcal{O} \longrightarrow \frac{1}{3 \mathcal{N}} \sum_{\substack{\text { equiv.wave } \\ \text { vectors } \boldsymbol{k}}}^{\mathcal{N}}\left[\left|\Phi_{\boldsymbol{k}}^{(a b ; c d)}\right|^{2}+\left|\Phi_{\boldsymbol{k}}^{(a c ; b d)}\right|^{2}+\left|\Phi_{\boldsymbol{k}}^{(a d ; b c)}\right|^{2}\right],
$$

where $\mathcal{N}$ is the number of equivalent wave vectors one can construct. This is a 4-replica quantity [see (4)], so 6 instantaneous overlaps are associated to each instance of the correlators. To define $\hat{q}$ we need to propose a function of the six overlaps in order to get a one-to-one correspondence.

Let us reorder each 6-plet of instantaneous overlaps $\left\{q^{(i j)}\right\}$ in the form of six sorted overlaps $\left\{q_{k}\right\}$

$$
\left\{q^{(a b)}, q^{(a c)}, q^{(a d)}, q^{(b c)}, q^{(b d)}, q^{(c d)}\right\} \longrightarrow\left\{q_{1} \leq q_{2} \leq q_{3} \leq q_{4} \leq q_{5} \leq q_{6}\right\} .
$$

The following are natural test conditioning variates:

$$
\hat{q}=\left\{\begin{array}{lrl}
q_{\min }=q_{1} & \text { (the minimum) } \\
q_{\max }=q_{6} & \text { (the maximum) } \\
q_{\text {med }}=\frac{1}{2}\left(q_{3}+q_{4}\right) & \text { (the median) } \\
q_{\text {av }}=\frac{1}{6}\left(q_{1}+q_{2}+q_{3}+q_{4}+q_{5}+q_{6}\right) & \text { (the average) } .
\end{array}\right.
$$

table 2 depicts the $c_{1}$ and $c_{2}$ terms, and their ratio, for all the conditioning variates, for a single triplet $(T, L, h)$ and $\boldsymbol{k}=(0,0,0)$. The best conditioning variate is clearly the median, since it has the highest $c_{2} / c_{1}$ ratio. The situation is similar for other choices of $(T, L, h)$.

For a qualitative description of the difference between the diverse conditioning variates, in figure 5 (top) the reader can appreciate the probability distribution functions for each of the conditioning variates, while in figure 5 (bottom) we plotted the conditional 
susceptibilities. From (18) we stress that the integral of the values on the top times the values of the bottom set yields the average susceptibility, which is indicated with a horizontal line on the bottom plot of figure 5. As it is also reflected by table 2, $q_{\max }$

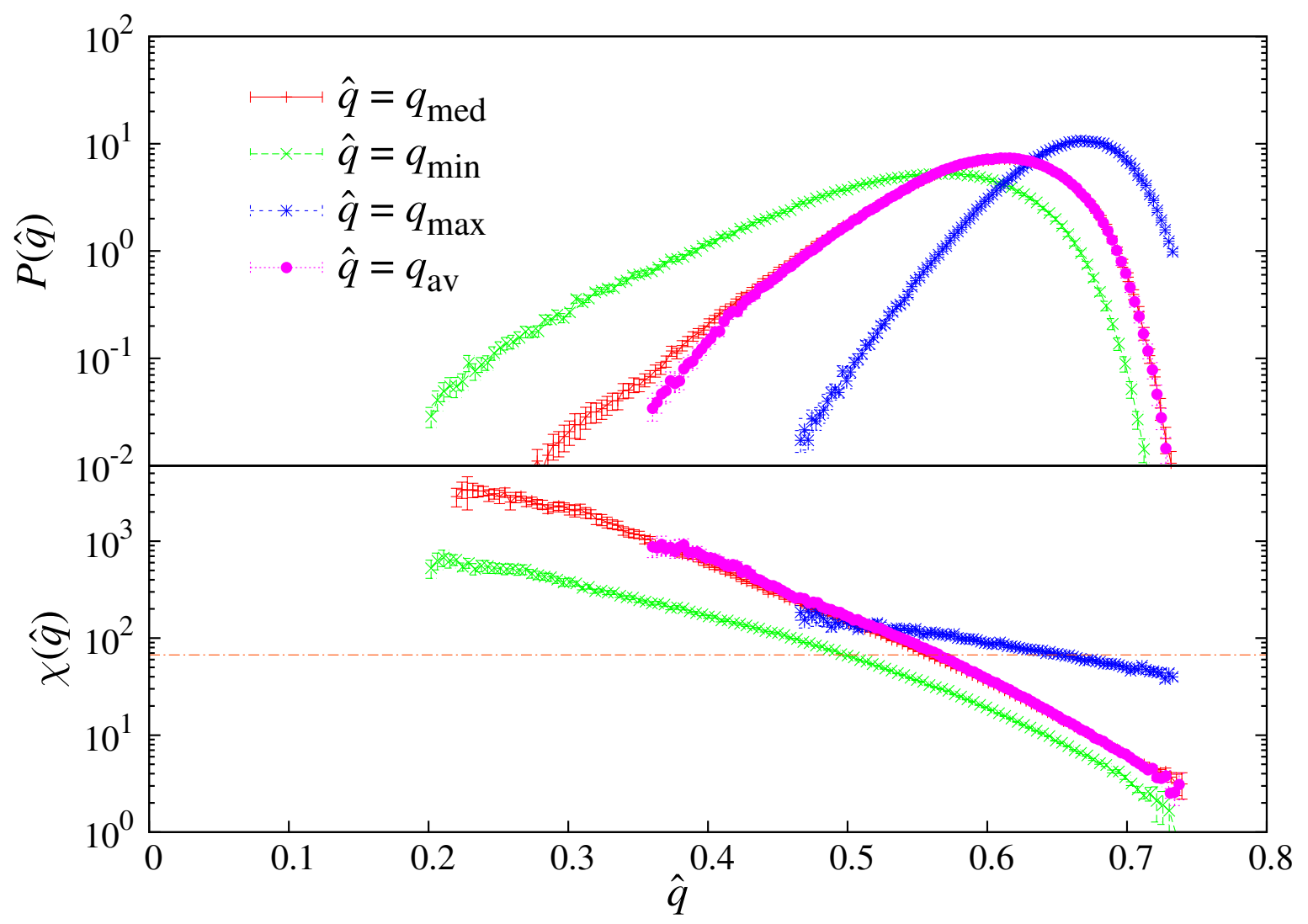

Figure 5. Features of the diverse conditioning variates we proposed for $L=32$, $h=0.2$ and $T=0.805128$. The top figure shows the histograms $P(\hat{q})$ for our four candidates of conditioning variate: the minimum overlap $q_{\min }$ [of the six we can make with four replicas, recall [23]], the maximum $q_{\max }$, the median $q_{\text {med }}$ and the average $q_{\mathrm{av}}$. The histograms were constructed as explained in Appendix C. The bottom figure depicts the size of the susceptibility $\chi$ for each value of the conditioning variate. The horizontal line marks the value of $\chi$ when it is averaged over the full set of measurements. For aesthetic reasons in both figures we have cut the curves at the two end points, where they become extremely noisy due to poor sampling.

is the worst conditioning variate, as its $\chi$ does not vary much with the fluctuations of $q_{\text {max }}$. The steepest slope is obtained when the conditioning variate is $q_{\text {av }}$ or $q_{\text {med }}$, but the latter is smoother and covers a wider range of $\chi$.

Figure 5 also displays the large deviations present in the system. In fact one can see that the value of $q_{\text {med }}$ at which the $P\left(q_{\text {med }}\right)$ has its maximum is significantly different with respect to the value of $q_{\text {med }}$ at which $\chi\left(q_{\text {med }}\right)$ assumes the value of the average. 


\section{Quantiles and a modified finite-size scaling ansatz}

We stated in section 2 that the set of measurements with low $\hat{q}$ has a very different behaviour from the measurements with high $\hat{q}$ (recall figure3). From now on, we shall restrict ourselves to $\hat{q}=q_{\text {med }}$, since we evinced that the median is our best conditioning variate. Our next goal will be to carry out a finite-size scaling analysis based on the $P\left(q_{\text {med }}\right)$ that lets us observe different parts of the spectrum of behaviours of the system.

In order to analyse separately these different sets of measures, we divide the $P\left(q_{\text {med }}\right)$ in 10 sectors, each containing $10 \%$ of the measured $q_{\text {med }}$. We focus our analysis on the values of $q_{\text {med }}$ that separate each of these sectors. They are called quantiles (see, e.g., [70]), and we label them with the subscript $i=1, \ldots, 9$. If we call $\tilde{q}_{i}(h, T, L)$ the value of the $i^{\text {th }}$ quantile, we can define it in the following implicit way:

$$
\int_{-1}^{\tilde{q}_{i}} \mathrm{~d} \hat{q} P(\hat{q})=\frac{i}{10}
$$

In Appendix C we explain how $\tilde{q}_{i}(h, T, L)$ was computed.

We can adapt to the $i^{\text {th }}$ quantile the definitions we gave in section 4 :

$$
\begin{aligned}
\chi_{\mathrm{R}, i}(\boldsymbol{k}) & =\frac{1}{V} E\left(\left|\hat{\Phi}_{k}^{(a b ; c d)}\right|^{2} \mid \tilde{q}_{i}\right), \\
\xi_{L, i} & =\frac{1}{2 \sin \left(k_{\min } / 2\right)} \sqrt{\frac{\chi_{\mathrm{R}, i}(\mathbf{0})}{\chi_{\mathrm{R}, i}(2 \pi / L, 0,0)}-1}, \\
R_{12, i} & =\frac{\chi_{\mathrm{R}, i}(2 \pi / L, 0,0)}{\chi_{\mathrm{R}, i}(2 \pi / L, \pm 2 \pi / L, 0)} .
\end{aligned}
$$

This way we can extend the finite-size scaling methodology to the $i^{\text {th }}$ quantile:

$$
\left.\frac{\xi_{L}}{L}\right|_{T, h, L, i}=\mathcal{F}_{\xi_{i}}\left(L^{1 / \nu}\left(T-T_{\mathrm{c}}\right)\right) \quad,\left.\quad R_{12}\right|_{T, h, L, i}=\mathcal{F}_{R_{i}}\left(L^{1 / \nu}\left(T-T_{\mathrm{c}}\right)\right) .
$$

This is a new approach for finite-size scaling. Although it demands a very large amount of data because it is done over a small fraction of the measurements, it allows us to perform finite-size scaling on selected sets of measurements. Let us stress that no $a$ priori knowledge is required on the probability distribution function $P\left(q_{\text {med }}\right)$ : Quantiles are conceived in order to define a scaling that self-adapts when the volume increases. In Appendix A.2 we show the validity of this ansatz in zero field.

\subsection{The $P\left(q_{\mathrm{med}}\right)$}

Up to our knowledge, despite its simplicity the median overlap $q_{\text {med }}$ has not been studied before. In fact, we just lacked the motivation to investigate its features. Yet, now we base our analysis on this quantity, so we feel that it is necessary to dedicate it a paragraph.

By its definition, the probability distribution $P\left(q_{\text {med }}\right)$ of the median overlap has narrower tails than $P(q)$ (recall figure 2), although from figure 5 (top) it is clear that the strong fluctuations persist also with $q_{\text {med }}$. 
The median of $P\left(q_{\text {med }}\right)$ corresponds to the fifth quantile. We will prefer to call it " $5^{\text {th }}$ quantile" rather than "median of the median overlap". Of the nine studied quantiles it is the smoothest and has the least finite-size effects, as one can see from figure 6 (inset). Further analysis is given in Appendix E.

We remark also that the separation between the different $\tilde{q}_{i}$ 's can be used as order parameter, since its thermodynamic limit should be zero in the paramagnetic phase, and greater than zero in the possible low-temperature phase due to the (would-be) replica symmetry breaking. Figure 6 shows the difference between the $8^{\text {th }}$ and the $2^{\text {nd }}$ quantile, i.e., the $q_{\text {med }}$-span of the central $60 \%$ of the data. If we were able to extrapolate a clean $L \rightarrow \infty$ limit for this curve, we would be able to answer to whether the transition exists or not. Unfortunately, even for $T>T_{\mathrm{c}}(h=0)=1.1019(29)$, where we know that we are in the paramagnetic phase, it is not possible to make good extrapolations since the trend is strongly non-linear. In Appendix E we show that extrapolations to the thermodynamic limit were only possible in the trivial case of $h=0.4$ (deep paramagnetic phase), and that between all the quantiles, the median curve is the one that shows less finite-size effects.

\section{Results}

As already stressed, the behaviour of the system is characterised by very strong fluctuations, and a wide and asymmetric $P(q)$. As a result, the average and median behaviour are very different. In figure 7, we show the replicon susceptibility: its average $\chi$ on the left plot, and its fifth quantile $\chi_{5}$. Motivated by the arguments in section 6 all the quantiles we show in this section use the conditioning variate $\hat{q}=q_{\text {med }}$.

Visibly, not only is the average susceptibility much larger than the $5^{\text {th }}$ quantile, but also the two have peaks at different temperatures. Also, finite-size effects are much stronger in the case of $\chi_{5}$ (yet, recall the inset in figure 6, finite-size effects on $\tilde{q}_{5}$ are tiny)

We show in figure 8 how sorting the data with the quantiles revealed the presence of different types of behaviour, by plotting the $\xi_{L} / L$ and the $R_{12}$ for quantiles 1,5 and 9 at $h=0.2$ There are two vertical lines in each figure. The one on the left represents the upper bound $T^{\mathrm{up}}(h)$ for the phase transition (meaning that no phase transition can occur for $\left.T>T^{\mathrm{up}}(h)\right)$ given in [34], while the one on the right indicates the zero field critical temperature $T_{\mathrm{c}}=1.1019(29)$ [51].

We can see that the $1^{\text {st }}$ quantile has the same qualitative behaviour of the average (figure 1), but lower values, since the main contribution to the average comes from data whose $q_{\text {med }}$ is even lower than $\tilde{q}_{1}$. Moreover, one can notice that in figure 1 the indicators

$\|$ We made power law extrapolations to $L \rightarrow \infty$ of the maxima of the susceptibility, but they were not satisfactory (too large $\chi^{2} / \mathrm{DOF}$ ). Only for $h=0.2,0.4$ were we able to fit the maxima's heights and obtained $\eta(h=0.2) \approx 0.6$ and $\eta(h=0.4) \approx 0.9$.

I In Appendix A.1 we show the same plots for $h=0.1$, and in Appendix A.2 we validate our method in the null-field case, where it is known that there actually is a phase transition. 


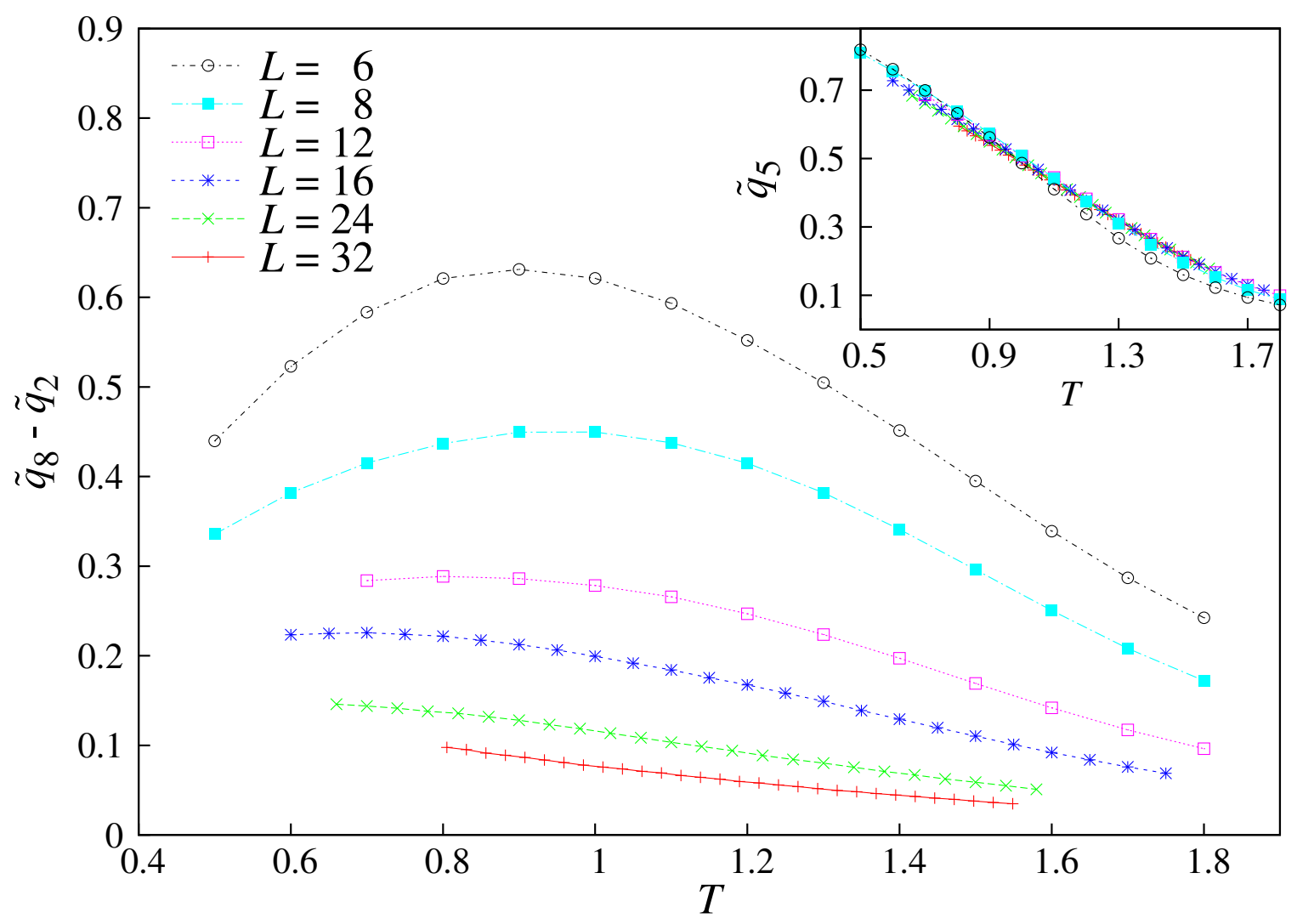

Figure 6. Using $q_{\text {med }}$ as conditioning variate, we show the temperature dependence of the difference between quantiles $\tilde{q}_{8}-\tilde{q}_{2}$, for all our lattice sizes, in a field of intensity $h=0.2$. This corresponds to the width of the central $60 \%$ of area of $P\left(q_{\mathrm{med}}\right)$. This quantity can reveal a phase transition, since in the paramagnetic phase the $P\left(q_{\text {med }}\right)$ should be a delta function, while in the spin-glass phase it should have a finite support. We show the central $60 \%$ and not a wider range because it is an equivalent indicator of the phase transition, and it is safer from rare events that would vanish in the thermodynamic limit. In the inset we show the position of $5^{\text {th }}$ quantile as a function of temperature in all our lattice sizes. It is a very smooth curve with very small finite-size effects.

$\xi_{L} / L$ and $R_{12}$ show a different qualitative behaviour when the lattices are small $\left(R_{12}\right.$ shows a crossing). This discrepancy vanishes when we look only at the first quantile: Separating different behaviours enhances the consistency between $\xi_{L} / L$ and $R_{12}$.

The behaviour of the $5^{\text {th }}$ quantile is quite different, since now it appears reasonable that the curves cross at some $T \lesssim T^{\mathrm{up}}(h)$. The crossings become even more evident when we consider the highest quantile.

All this is consistent with the arguments of section 2, where we showed how the correlation function is dominated by a little portion of data, near the first quantile (figure 3), while the behaviour of the majority of the samples is hidden.

Unfortunately, the high non-linearity of the curves impedes an extrapolation of the crossing points, but they are apparently compatible with the upper bound $T^{\mathrm{up}}$, and their heights apparently do not depend on the intensity of the applied field $h$ Appendix 


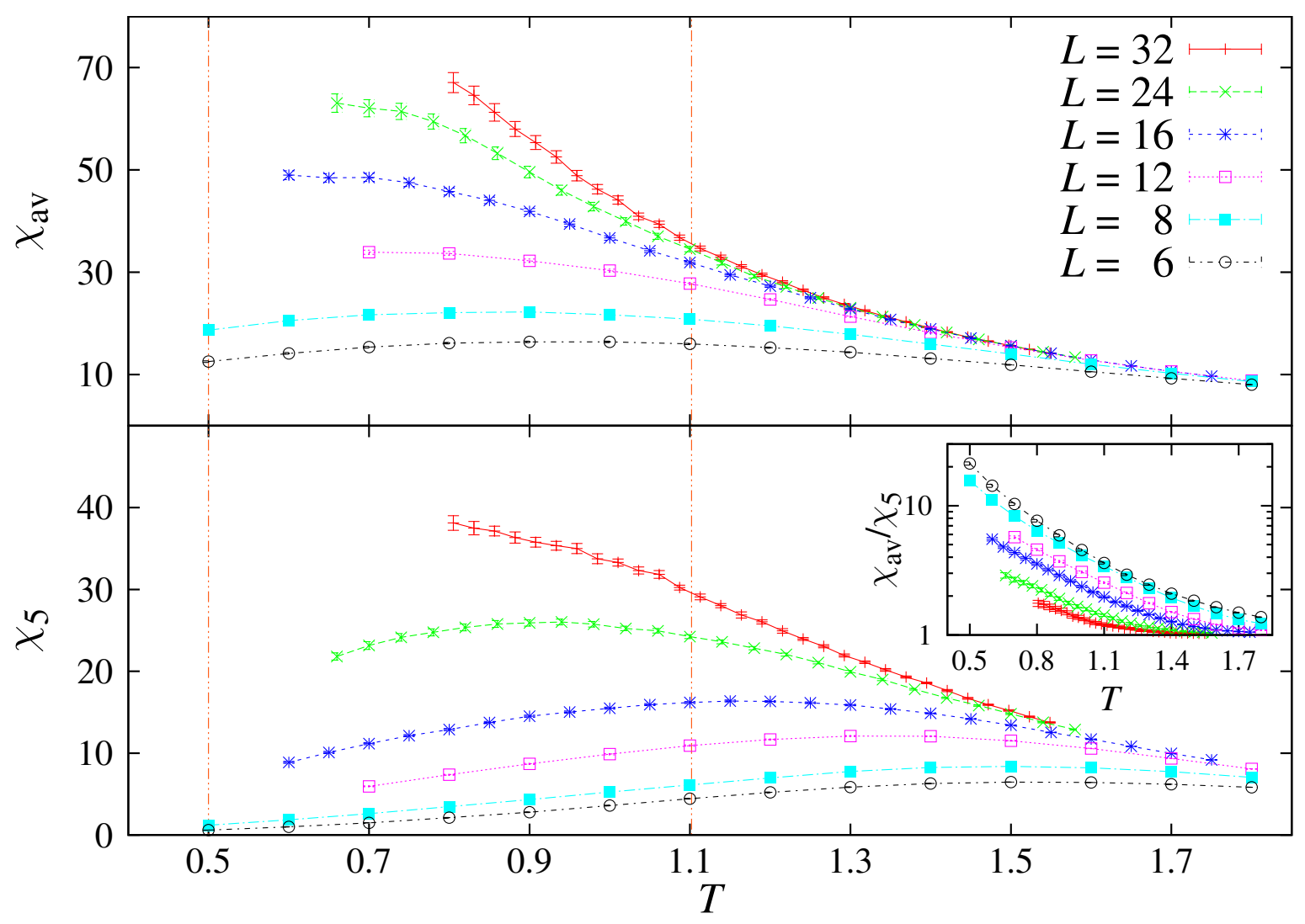

Figure 7. The replicon susceptibility $\chi$ as a function of the temperature, for all the simulated lattice sizes and the field $h=0.2$. We represent its average $\chi$ (top), and the $5^{\text {th }}$ quantile $\chi_{5}$ with $\hat{q}=q_{\text {med }}$ (bottom). In both plots, the two vertical lines represent the upper bound of the possible phase transition $T^{\mathrm{up}}(h=0.2)=0.5$ [34], and the zero-field critical temperature $T_{\mathrm{c}}(h=0)=1.109(29)$ [51. The amplitudes and the positions of the peaks of $\chi$ are strikingly different (mind the different scales in the $y$ axes). The inset shows the ratio between the two, which we expect to tend to an order one constant in the thermodynamic limit. This is actually what we see at high temperatures.

A.

\section{This is not an echo of the $h=0$ transition}

The crossing suggested by the quantiles 5 and 9 in figure 8 is unlikely to be caused by the zero-field transition, since it appears at $T<T_{\mathrm{c}}$, and shifts towards lower temperatures as the lattice size increases. Also, the value of $\xi_{L} / L\left(R_{12}\right)$ at the possible crossing point of the fifth quantile is upper-bounded to $\xi_{L} / L \simeq 0.16\left(R_{12} \simeq 1.65\right)$, while for $h=0$ it is considerably larger $\left(\xi_{L}\left(T_{\mathrm{c}}\right) / L \simeq 0.28\left[R_{12}\left(T_{\mathrm{c}}\right) \simeq 2.15\right]\right)$, see Appendix A.2. In this section we will give more arguments sustaining that what is seen is not an effect of the zero-field transition. 


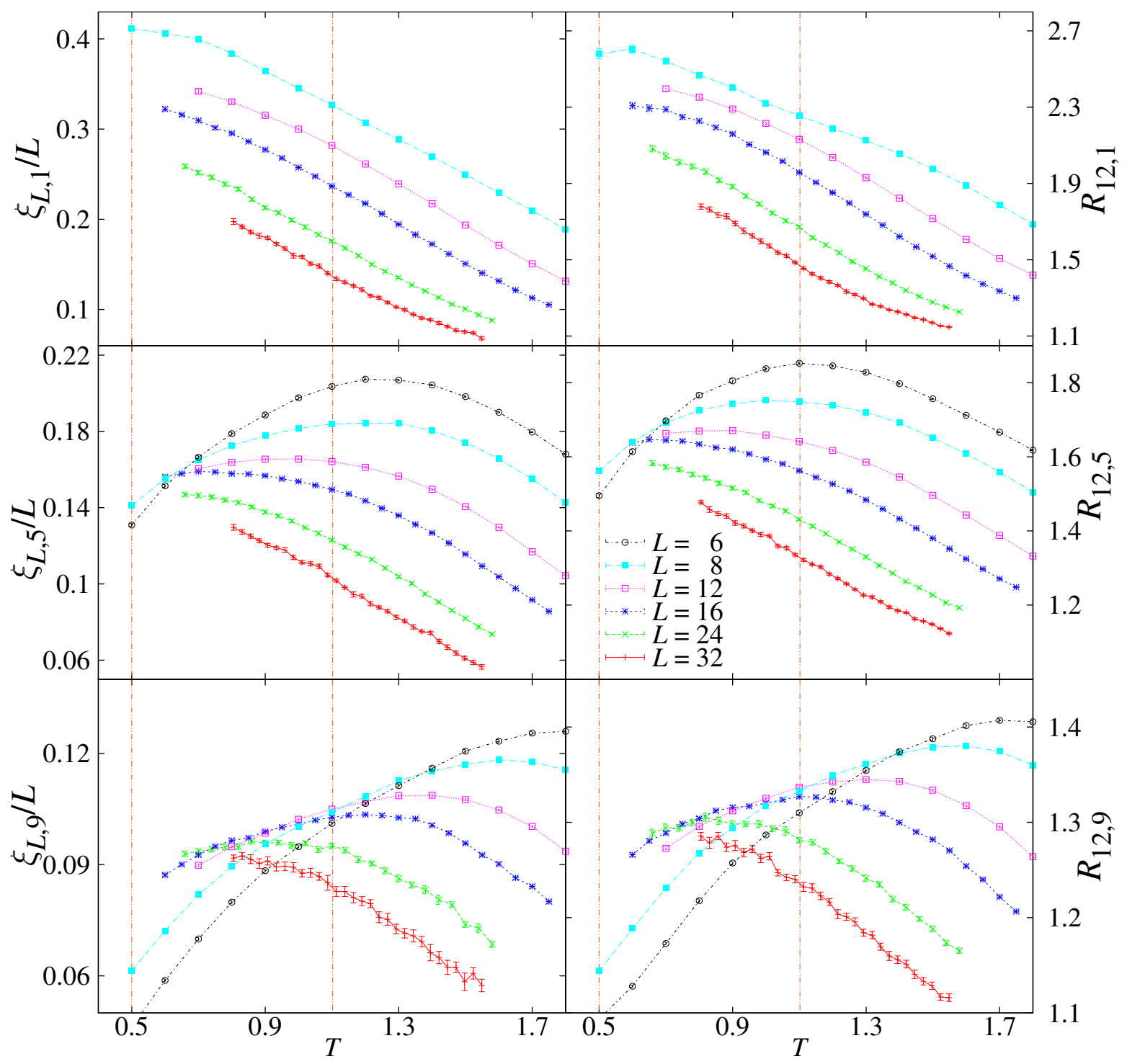

Figure 8. Finite-size indicators of a phase transition, computed for $h=0.2$. On the left side we plot, for quantiles 1 (top), 5 (middle) and 9 (bottom), the correlation length in units of the lattice size $\xi_{L} / L$ (left) versus the temperature, for all our lattice sizes except $L=6$ (we show in Appendix D that the quantile description is not suitable for $L=6$ because there is a double peak in the $P(q)$ ). On the right we show analogous plots, for $R_{12}$. The vertical line on the left marks the upper bound $T^{\text {up }}$ for a possible phase transition given in [34, while the one on the right marks the zero-field transition temperature $T_{\mathrm{c}}$ given in [51]. Quantile 1 has the same qualitative behaviour of the average $\xi_{L} / L$, shown in figure 1 , while quantiles 5 and 9 suggest a scale invariance at some temperature $T_{h}<T^{\text {up }}$.

\subsection{An escaping transition}

As pointed out in section 2, there is a controversy because we observe a wide $P(q)$, just like in the mean-field model, but the curves $\xi_{L} / L(T)$ and $R_{12}(T)$ do not show any sign of 
a crossing. If we were in the presence of a phase transition, a straightforward explanation could reside in an anomalous exponent $\eta$ close to 2 [52, since at the critical temperature the replicon susceptibility scales as $\chi_{\mathrm{R}}(L) \sim L^{2-\eta} \sqrt{15}$. It is possible to calculate $\eta$ with the quotients method [48, 67], by comparing the susceptibility of different lattice sizes at the critical point $T^{*}$ :

$$
\frac{\chi_{2 L}\left(T^{*}\right)}{\chi_{L}\left(T^{*}\right)}=2^{2-\eta}+\ldots,
$$

where the dots stand for subleading terms. This definition only makes sense at criticality, but we can extend it in an effective manner to a generic temperature. This way we can delineate an effective exponent

$$
\eta_{\mathrm{eff}}(T ; L, 2 L)=2-\log _{2} \frac{\chi_{2 L}(T)}{\chi_{L}(T)} .
$$

In case there were a phase transition at a finite temperature $T_{h}$, we would have $\eta_{\text {eff }}\left(T_{h}\right)=\eta$. We should have $\eta_{\text {eff }}=2$ in the paramagnetic phase, $\eta_{\text {eff }}=-1$ in the deep spin-glass phase [see Appendix B.1, keeping in mind that $\eta_{\text {eff }}=-1$ is somewhat trivial in the limit $h \rightarrow 0$, where $\chi$ reduces to $\left.\chi=V E\left(q^{2}\right)\right]$ and signs of a crossing at $\eta_{\mathrm{eff}}=\eta(h=0)=-0.3900(36)$ [51] in the limit of a complete domination by the $h=0$ transition.

In figure 9 we show $\eta_{\text {eff }}(T)$ for $h=0.4, h=0.1$, and $h=0$ (the $h=0$ data come from the simulations we performed in [51]) ${ }^{\dagger}$ If a phase transition were present, but hidden by heavy finite-size effects, we would expect at least that the $L$-trend of $\eta_{\text {eff }}$ be decreasing. Contrarily, the larger our lattices, the wider the temperature range in which $\eta_{\text {eff }}=2$. The apparent phase transition shifts towards lower temperature when we suppress finite-size effects. The data in our possession is not enough to state whether this shift will converge to a positive temperature. In any case, this is compatible with the upper bounds to a possible transition given in [34].

On the other side, $\eta_{\text {eff }}$ stays positive for all our simulated lattices (except $h=0.05$, $L=6)$, and that even for $T<T_{\mathrm{c}}(h=0)$ it tends to some value around 0.5 , so it is unlikely that the null field transition is dominating the system's behaviour.

\subsection{Scaling at $T=T_{\mathrm{c}}(h=0)$}

From the scaling with the lattice linear size of $\xi_{L} / L$ at $T_{\mathrm{c}}=T_{\mathrm{c}}(h=0)$, we can get another element to discard the hypothesis that the $h=0$ transition is biasing significantly our measures. Assuming that there is no critical line for $h>0$, a very large correlation length could be due to an echo of the zero-field transition or a low-temperature effect. In a theory that predicts that system is critical only at $h=0, T=T_{\mathrm{c}}$, the effects of this echo on the $h>0$ behaviour should be maximal near $T=T_{\mathrm{c}}$. So, if we find a $\xi$ that is large compared to our lattice sizes for $T<T_{\mathrm{c}}$, a primary check is to monitor the scaling of the coherence length at $T_{\mathrm{c}}$. figure 10 shows the scaling of $\xi_{L} / L$ at $T_{\mathrm{c}}$ with

\footnotetext{
+ For each jackknife block we calculated $\eta_{\mathrm{eff}}(T)$ and made a cubic spline temperature interpolation.
} 


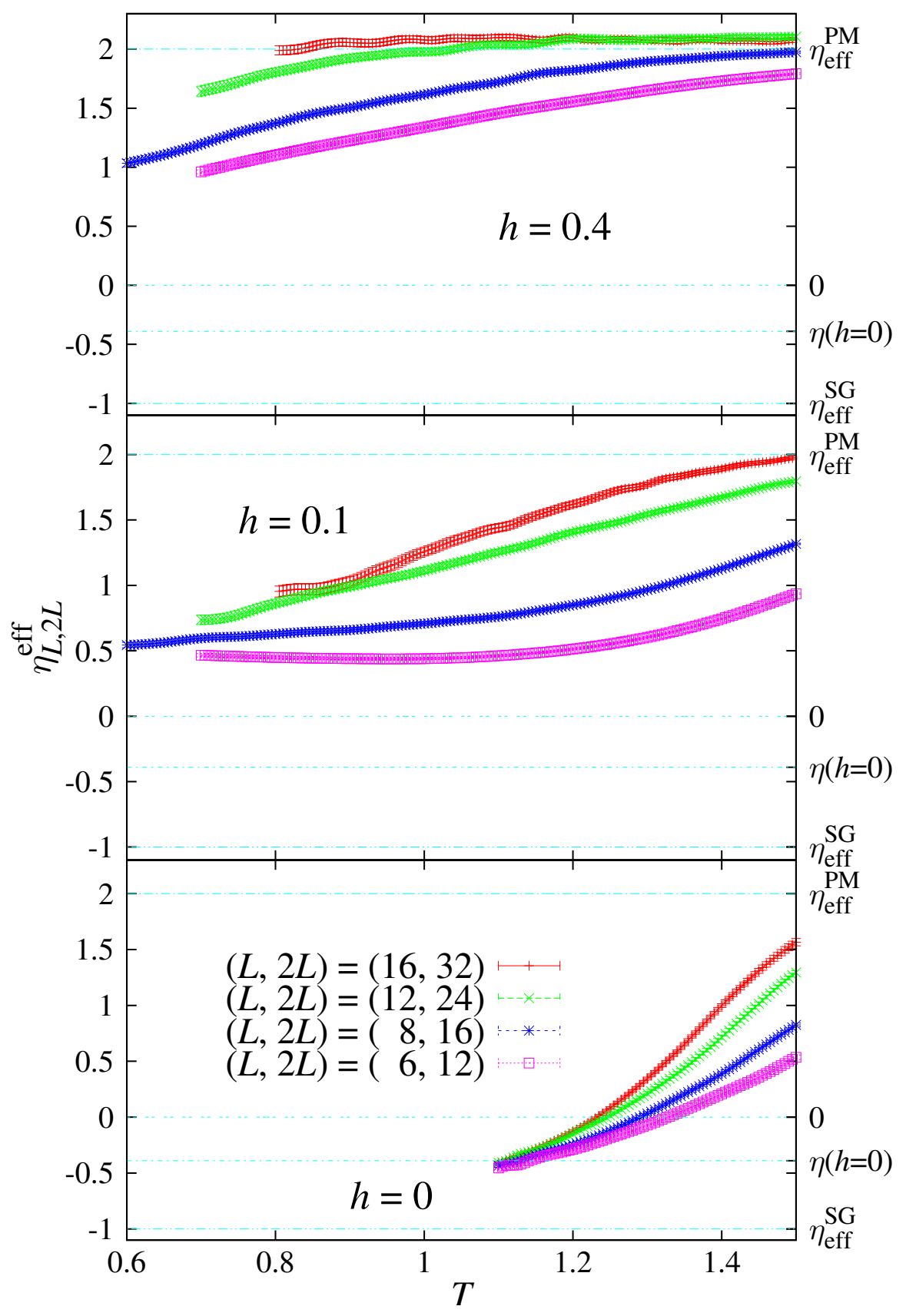

Figure 9. We plot $\eta_{\text {eff }}(T)$, defined in (30), for all the pairs $(L, 2 L)$ we could form. The magnetic fields are $h=0.4$ (top), $h=0.1$ (centre) and $h=0$ (bottom). The $h=0$ data comes from [51. In each plot we uses horizontal lines to underline meaningful limits, and we label them with a tic on the right axis. From up to down, we depict the limit $\eta_{\mathrm{eff}}^{\mathrm{PM}}=2$ of a system in the paramagnetic phase, the $\eta_{\mathrm{eff}}=0$ axis, the zero-field value $\eta_{\mathrm{eff}}\left(h=0, T_{\mathrm{c}}\right)=-0.3900(36)$ [51, and its value in a deep spin-glass phase $\eta_{\mathrm{eff}}^{\mathrm{SG}}=-1$. Notice the difference between the case with or without a field. For $h=0.1$ the curves appear to converge to a positive $\eta_{\text {eff }} \simeq 0.5$, while in the latter all the curves become negative and merge at $\eta_{\mathrm{eff}}\left(h=0, T_{\mathrm{c}}\right)$. 
$h=0.2$. We plot the average, the first, the fifth and the highest quantile. All of them show a clear decrease of $\xi_{L} / L$ when increasing the lattice size, so our lattice sizes are large enough to state that the divergence at $h=0$ is not dominating $\xi_{L}$ 's behaviour. On the other side, we are still far from the thermodynamic limit, since when the lattices are large enough, $\xi_{L}\left(T_{\mathrm{c}}\right) / L$ should decay to zero linearly in $1 / L$.

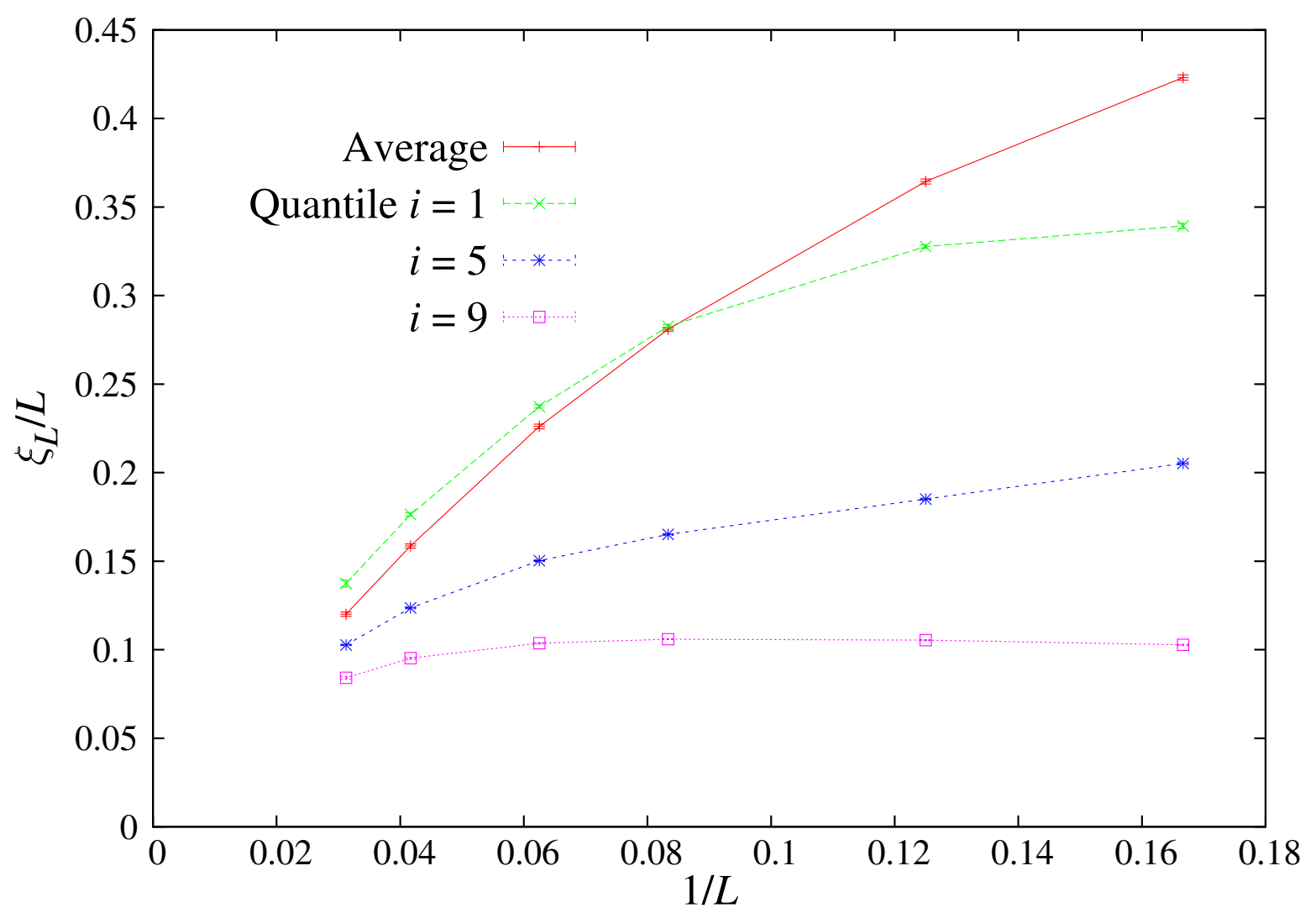

Figure 10. Scaling of $\xi_{L} / L$ at the null-field critical temperature $T_{\mathrm{c}}=1.109(29)$ [51], with $h=0.2$. We show the behaviour of the average, and of quantiles 1,5 and 9 . If $L$ is large enough, $\xi_{L} / L$ should go as $1 / L$, while if the system is seeing purely an echo of the divergence of the $h=0$ transition transition, then $\xi_{L} / L$ should be constant.

\section{Conclusions}

We have studied the equilibrium behaviour of the three-dimensional Ising EdwardsAnderson spin glass in an external magnetic field. Thermalising the system at sufficiently low temperature was a computationally hard task and required the use of the Janus dedicated computer to thermalise lattice sizes up to $L=32$, down to temperatures $T \geq 0.8$.

First of all, we carried out a traditional analysis of our data. We chose observables that would be scale invariant at the critical temperature, and compared them for different lattice sizes, looking for crossings in their temperature curves. With this procedure we found no traces of a phase transition. 
Yet, the scenario is more complicated. Despite the absence of crossings, indications that something non-trivial is going on are given by signals such as a growing correlation length (even for our largest lattices), peaks in the susceptibility, and a wide probability distribution function of the overlap.

We noticed a wide variety of behaviours within the same set of simulation parameters. Some measurements presented signs of criticality, while others did not. So, we tried to classify them in a meaningful way. We sorted our observables with the help of a conditioning variate, and came up with a quantitative criterion to select the best conditioning variate. Between the ones we proposed, the function of the instant overlaps that made the best conditioning variate turned out to be the median overlap $q_{\text {med }}$.

As a function of the median overlap, the scenario appeared rather non-trivial. The averages turned out to be dominated by a very small number of measurements. Those with a small $q_{\text {med }}$ behaved similarly to the average: long correlation lengths, very large susceptibilities, and no signs of criticality. On the other side, the median behaviour was far from the average, and the behaviour of most of the measurements was qualitatively different from the average, with smaller correlation lengths and susceptibilities, but nonnegligible indications of scale invariance right below the upper bound $T^{\mathrm{up}}(h)$ given in [34]. Furthermore, separating the different behaviours of the system we obtain mutually consistent indications of criticality from our primary dimensionless magnitudes $\xi_{L, i} / L$ and $R_{12, i}$. The achievement of this consistency is an important step forward with respect to [29], where the phase transition was revealed only by the $R_{12}$ indicator, but it was invisible to $\xi_{L} / L$.

Unfortunately we were not able to make a quantitative prediction on the critical temperatures $T_{\mathrm{c}}(h)$, because the observables as a function of the lattice size and of the temperature were very nonlinear, and the temperatures we reached were not low enough reliably to identify the crossing points of the quantile-dependent $\xi_{L, i} / L$ and $R_{12, i}$.

Overall, the presence of a phase transition appears plausible from our simulations (see also Appendix G). Perhaps more importantly, now the challenge is well defined: in order to be able to give, numerically, a conclusive answer on the presence of a de Almeida-Thouless line we need push our simulations down to $T \simeq 0.4$ (at $h=0.2)$. We believe that Janus II, the next generation of our dedicated computer [34, will be able to assume this challenge.

\section{Acknowledgments}

We thank M.A. Moore and J. Yeo for pointing out to us the potential usefulness of the Fisher-Sompolinsky scaling.

The Janus project has been partially supported by the EU (FEDER funds, No. UNZA05-33-003, MEC-DGA, Spain); by the European Research Council under the European Union's Seventh Framework Programme (FP7/2007-2013, ERC grant agreement no. 247328); by MINECO (Spain) (contracts FIS2012-35719-C02, FIS2010- 


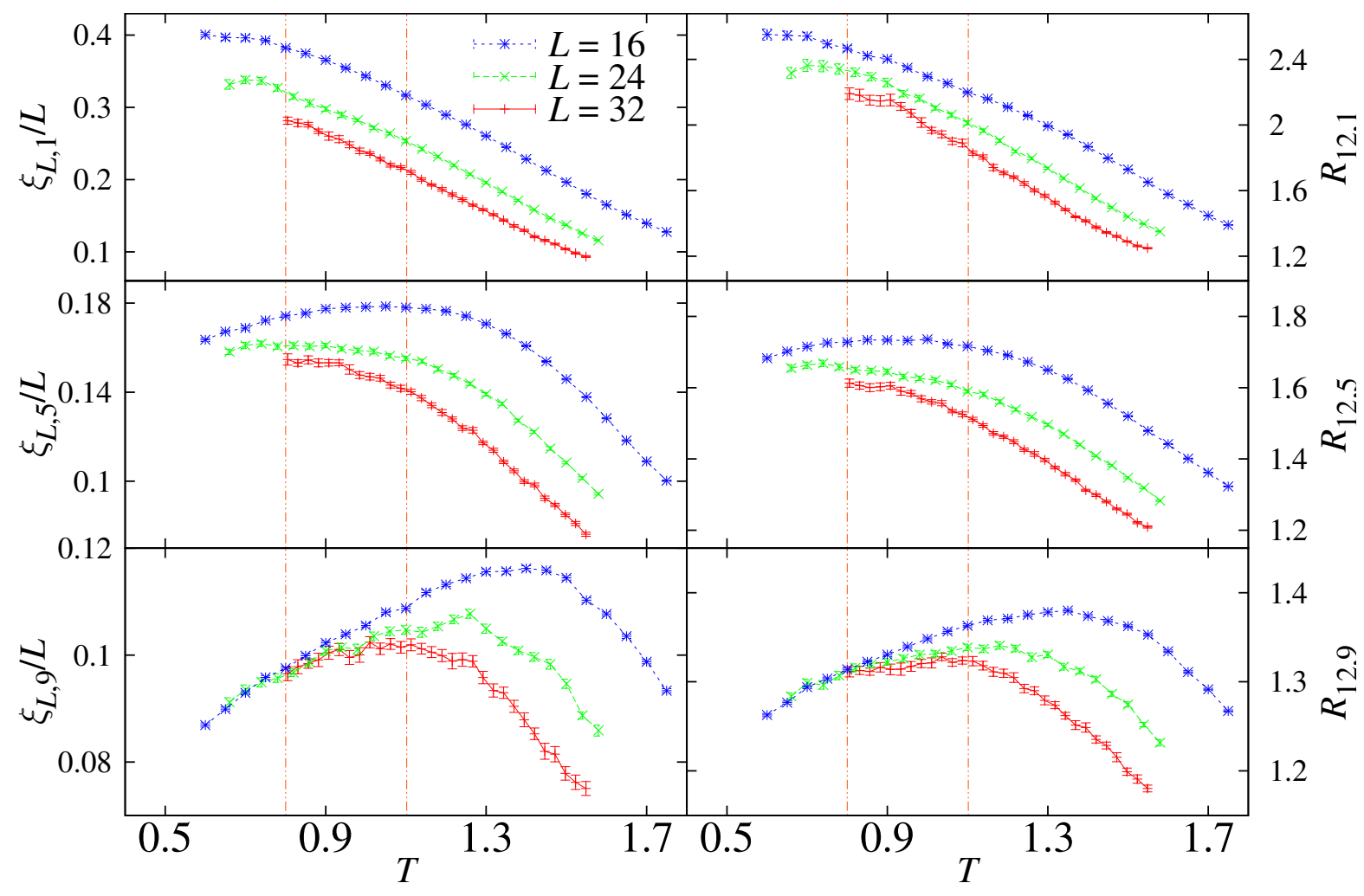

Figure A1. Same as figure 8, but for $h=0.1$. This time the effects of the zerotemperature transition are stronger, so we removed from the plot sizes $L=6,8,12$. In Appendix D we show that the quantile description is not suitable for smaller lattices due to crossover effects from the zero-field behavior.

16587, TEC2010-19207); by the SUMA project of INFN (Italy); by the Junta de Extremadura (GR10158); by the European Union (PIRSES-GA-2011-295302). F.R.T. was supported by the Italian Research Ministry through the FIRB project No. RBFR086NN1; M.B.-J. was supported by the FPU program (Ministerio de Educacion, Spain); R.A.B. and J.M.-G. were supported by the FPI program (Diputacion de Aragon, Spain); S.P.-G. was supported by the ARAID foundation; finally J.M.G.-N. was supported by the FPI program (Ministerio de Economia, Spain).

\section{Appendix A. Quantiles for different fields}

\section{Appendix A.1. Quantiles for $h=0.1$}

The careful reader might have noticed that the upper bound $T^{\mathrm{up}}(h)$ for the possible phase transition given in [34] is higher when the field is lower: $T^{\mathrm{up}}(0.1)=0.8>T^{\mathrm{up}}(0.2)$. It is then justified to ask oneself how do the quantile-plots look like for $h=0.1$. We show them in figure A1. Since the field is lower, the effects on the double peak on the first quantile Appendix D extend to larger lattices than for $h=0.2$. Thus, we show only the non-biased sizes, i.e., $L>12$. 
Although the $9^{\text {th }}$ quantile shows signs of scale invariance at $T=T^{\text {up }}(0.1)$, the behavior of the $5^{\text {th }}$ quantile suggests a scale invariance around $T=0.5$. We believe that the $5^{\text {th }}$ quantile is a better indicator, since the position of the fifth quantile $\tilde{q}_{5}$ has less finite-size effects (it practically has none, figure 6-inset) than $\tilde{q}_{9}$.

It is interesting to focus on the height of the crossings of each quantile, and compare them with $h=0.2$ (recall figure 8). This is expected to be a universal quantity, and in the hypothesis of a phase transition it should be the same for both fields. Although it is not possible to assign error bars to the these values, it is possible to see that both for $h=0.1$ and $h=0.2$ the heights are similar $\left(\xi_{L, 5} / L \approx 0.15, \xi_{L, 9} / L \approx 0.09, R_{12,5} \approx 1.6\right.$, $\left.R_{12,9} \approx 1.3\right)$.

\section{Appendix A.2. Quantiles for $h=0$}

We take advantage of our $h=0$ data from [51] to validate the quantile description by showing its behavior in the zero-field case. Two replicas would be enough to construct connected correlators in $h=0$, and using the 4-replica definitions proposed in section 4 only adds noise to the results. Yet, we opted for the latter option because the objective of the current section is the validation of the full procedure proposed herein.

In the absence of a magnetic field we expect that the curves $\xi / L(T)$ and $R_{12}$ cross no matter the quantile, since the behavior of the system is not dominated by extreme events and crossover fluctuations. Also, in this case the data in our hands arrive down to the critical point, so the crossings ought to be visible.

In fact, one can see from figure A2 that all the quantiles show visible signs of a crossing at $T_{\mathrm{c}}$ both in the case of $\xi_{L} / L$ and of $R_{12}$. Furthermore, if we plot the same data as a function of the scaling variable $L^{1 / \nu}\left(T-T_{\mathrm{c}}\right) / T_{\mathrm{c}}$ the data collapses well for all the quantiles (figure A2, insets).

Some reader may be surprised that quantiles 1 and 9 show different behavior, being $P(q)$ symmetrical (figure A3). The reason is that, although $P(q)$ is symmetrical, $P\left(q_{\text {med }}\right)$ is not. In fact, given six overlaps $q^{a b}, q^{a c}, q^{a d}, q^{b c}, q^{b d}, q^{c d}$ coming from four configurations $\left\{\sigma^{(a)}\right\},\left\{\sigma^{(b)}\right\},\left\{\sigma^{(c)}\right\},\left\{\sigma^{(d)}\right\}$, each enjoying a $Z_{2}$ symmetry, the distribution of their median privileges negative values. ${ }^{*}$ We show this in figure A3, where we give both the $P(q)$ and the $P\left(q_{\text {med }}\right)$ for $h=0, L=32, T=1.1$. The first is symmetrical and the second is not. To convince the reader that the starting configurations do enjoy $Z_{2}$ symmetry, we also construct the symmetrised functions $P^{(\mathrm{sym})}(q)$ and $P^{(\mathrm{sym})}\left(q_{\mathrm{med}}\right)$. These two functions are obtained by explicitly imposing the $Z_{2}$ symmetry: for each measurement we construct the $2^{4}$ overlaps with both $\left\{\sigma_{i}\right\}$ and $\left\{-\sigma_{i}\right\}$. It is visible from figure A3

* Let us give a simple example. Take $4 Z_{2}$-symmetric single-spin systems that can assume different values $s_{1}= \pm 1, s_{2}= \pm 2, s_{3}= \pm 3, s_{4}= \pm 4$. We can construct 6 overlaps $q_{i j}\left(s_{1}, s_{2}, s_{3}, s_{4}\right)$. If we explicitate the $Z_{2}$ symmetry, taking all the combinations of our random variables, the histogram of $q$ will be symmetric with zero mean. Yet, if we take the histogram of the median overlap, it will be asymmetric with mean $\left\langle q_{\text {med }}\right\rangle=-3$. This can easily be checked by computing all the possible combinations of the signs of the $s_{i}$ and computing the median in each case: $q_{\text {med }}(+1,+2,+3,+4)=5$, $q_{\text {med }}(+1,+2,+3,-4)=-1, q_{\text {med }}(+1,+2,-3,-4)=-3.5$, and so on. 


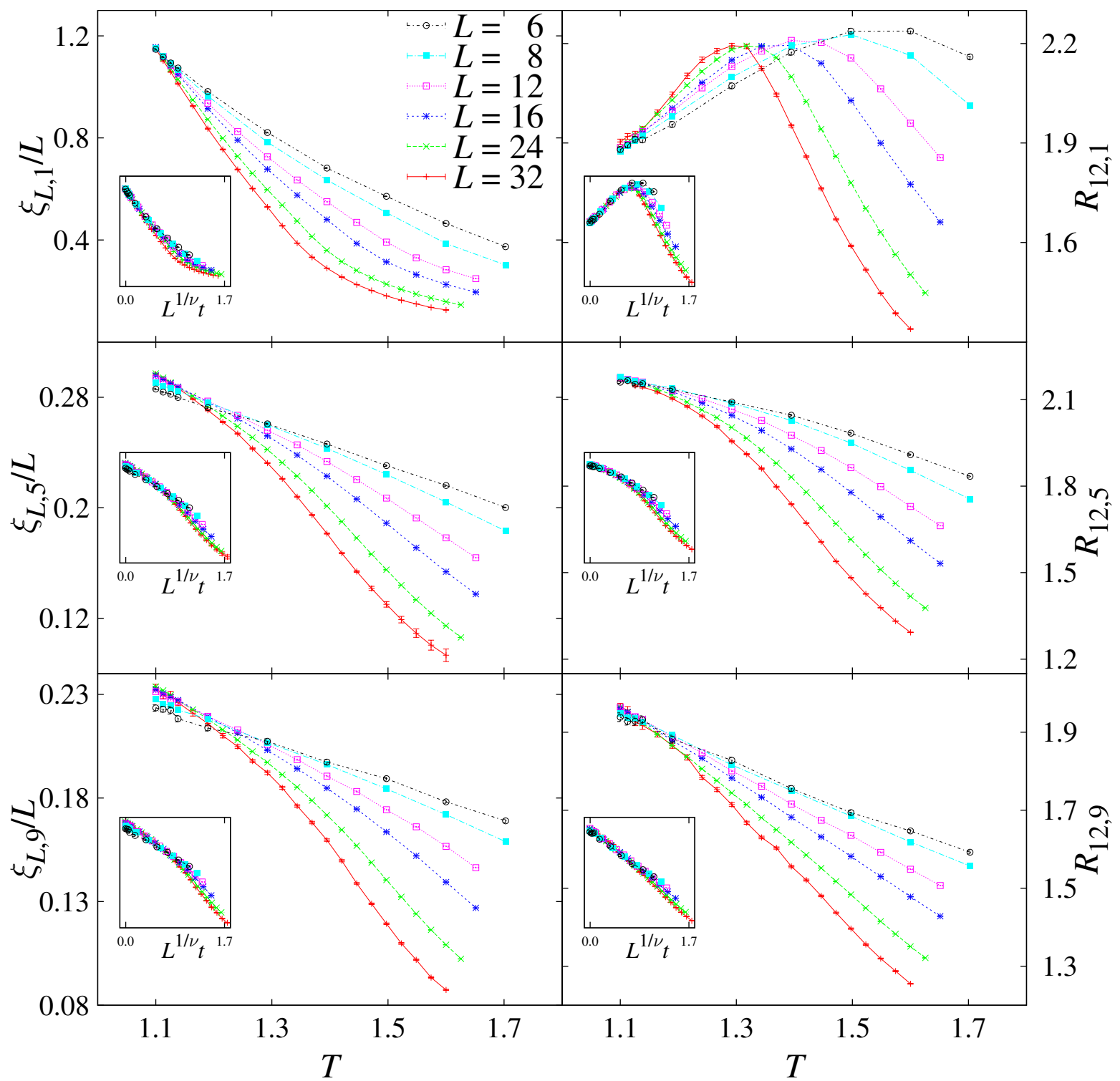

Figure A2. Same as figure 8, but for $h=0$. The data come from [51. We used 256000 samples for each lattice size. The insets show the same data of the larger sets, but as a function of the scaling variable $L^{1 / \nu} t$, where $t$ is the reduced temperature $t=\left(T-T_{\mathrm{c}}\right) / T_{\mathrm{c}}$.

that $P^{\text {(sym) }}\left(q_{\text {med }}\right)$ is asymmetric even though we imposed by hand the $Z_{2}$ symmetry on the configurations.

\section{Appendix B. 4-Replica Correlators}

In the presence of a magnetic field it is not possible to construct connected correlation functions with the use of only two replicas because in the paramagnetic phase $\overline{\langle q\rangle}=q_{\mathrm{EA}}>0$. 


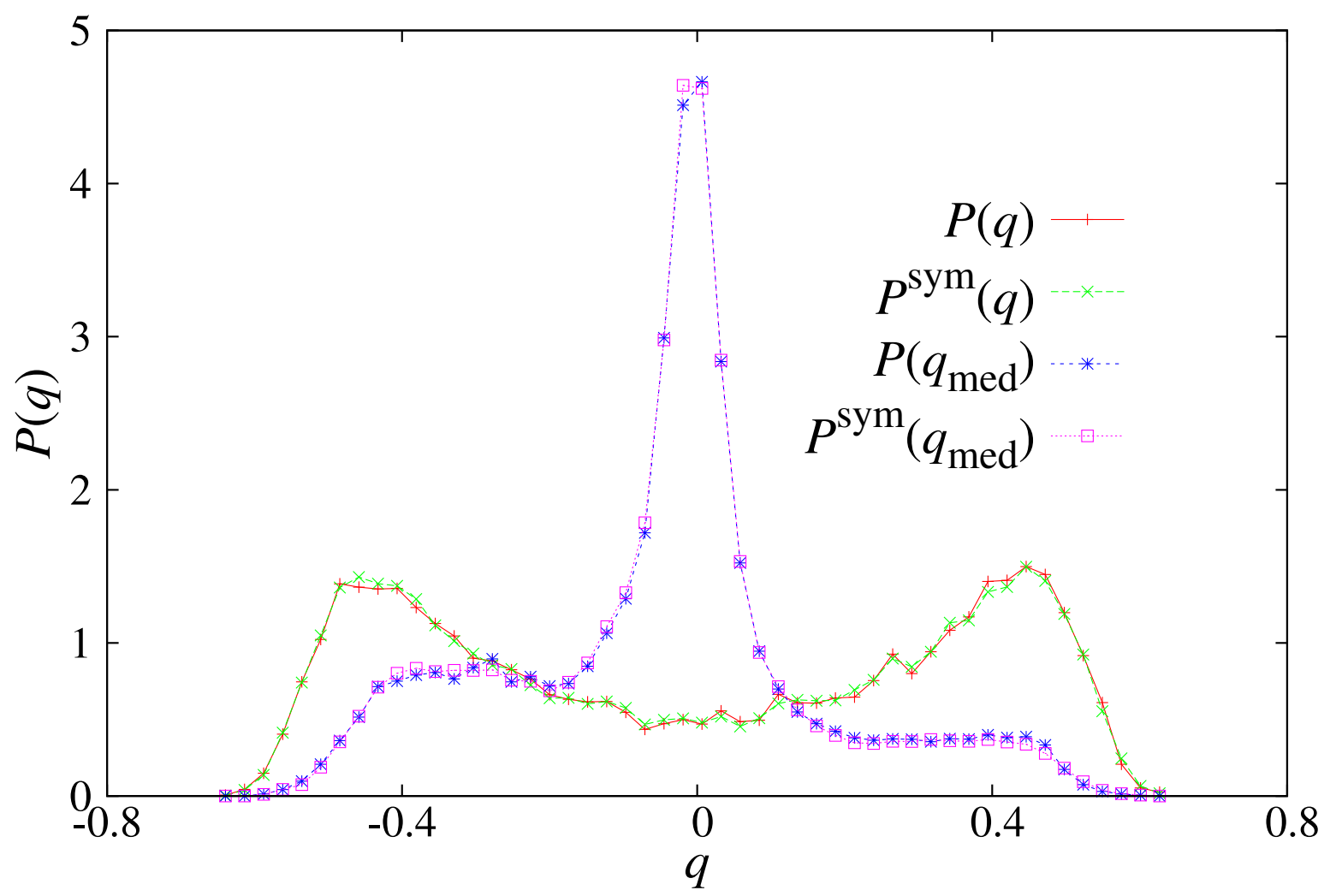

Figure A3. Probability distribution functions for $h=0, L=24, T=1.1$. The data come from 512 samples where we took all the $16^{4}$ combinations of overlaps per sample. We show $P(q)$, that in null field is symmetric, and $P\left(q_{\text {med }}\right)$, that is not. We also plot the symmetrised histograms $P^{(\mathrm{sym})}(q)$ and $P^{(\mathrm{sym})}\left(q_{\mathrm{med}}\right)$.

With 4 replicas we can construct 3 different correlators at distance $\boldsymbol{r}$.

$$
\begin{aligned}
G_{1}(\boldsymbol{r}) & =\frac{1}{V} \sum_{\boldsymbol{x}} \overline{\left\langle\sigma_{\boldsymbol{x}} \sigma_{\boldsymbol{x}+\boldsymbol{r}}\right\rangle^{2}} \\
& =\frac{1}{V} \sum_{\boldsymbol{x}} \overline{\left\langle\sigma_{\boldsymbol{x}}^{(a)} \sigma_{\boldsymbol{x}+\boldsymbol{r}}^{(a)} \sigma_{\boldsymbol{x}}^{(b)} \sigma_{\boldsymbol{x}+\boldsymbol{r}}^{(b)}\right\rangle} \\
G_{2}(\boldsymbol{r}) & =\frac{1}{V} \sum_{\boldsymbol{x}} \overline{\left\langle\sigma_{\boldsymbol{x}} \sigma_{\boldsymbol{x}+\boldsymbol{r}}\right\rangle\left\langle\sigma_{\boldsymbol{x}}\right\rangle\left\langle\sigma_{\boldsymbol{x}+\boldsymbol{r}}\right\rangle} \\
& =\frac{1}{V} \sum_{\boldsymbol{x}} \overline{\left\langle\sigma_{\boldsymbol{x}}^{(a)} \sigma_{\boldsymbol{x}+\boldsymbol{r}}^{(a)} \sigma_{\boldsymbol{x}}^{(b)} \sigma_{\boldsymbol{x}+\boldsymbol{r}}^{(c)}\right\rangle} \\
G_{3}(\boldsymbol{r}) & =\frac{1}{V} \sum_{\boldsymbol{x}} \frac{\left.1 \sigma_{\boldsymbol{x}}\right\rangle^{2}\left\langle\sigma_{\boldsymbol{x}+\boldsymbol{r}}\right\rangle^{2}}{\overline{\left\langle\sigma_{\boldsymbol{x}}^{(a)} \sigma_{\boldsymbol{x}+\boldsymbol{r}}^{(b)} \sigma_{\boldsymbol{x}}^{(c)} \sigma_{\boldsymbol{x}+\boldsymbol{r}}^{(d)}\right\rangle} .} \\
& =\frac{1}{V} \sum_{\boldsymbol{x}}
\end{aligned}
$$

None of those goes to zero for large $\boldsymbol{r}$, but, in the paramagnetic phase, for large $r$ they all tend to the same value, $q_{\mathrm{EA}}$. So, to create connected correlators, we can make two linearly independent combinations of them, and obtain the basic connected 
propagators of the replicated field theory [71] $甘$

$$
\begin{aligned}
& G_{\mathrm{R}}=G_{1}-2 G_{2}+G_{3}, \\
& G_{\mathrm{L}}=G_{1}-4 G_{2}+3 G_{3} .
\end{aligned}
$$

It is easy to check that these relations imply

$$
\begin{aligned}
& G_{\mathrm{R}}(\boldsymbol{r})=\overline{\left[\left\langle\sigma_{\boldsymbol{x}} \sigma_{\boldsymbol{x}+\boldsymbol{r}}\right\rangle-\left\langle\sigma_{\boldsymbol{x}}\right\rangle\left\langle\sigma_{\boldsymbol{x}+\boldsymbol{r}}\right\rangle\right]^{2}}, \\
& G_{\mathrm{L}}(\boldsymbol{r})=2 G_{\mathrm{R}}(\boldsymbol{r})-\Gamma_{2}(\boldsymbol{r}), \quad \Gamma_{2}(\boldsymbol{r})=\overline{\left[\left\langle\sigma_{\boldsymbol{x}} \sigma_{\boldsymbol{x}+\boldsymbol{r}}\right\rangle^{2}-\left\langle\sigma_{\boldsymbol{x}}\right\rangle^{2}\left\langle\sigma_{\boldsymbol{x}+\boldsymbol{r}}\right\rangle^{2}\right]} .
\end{aligned}
$$

The definitions in (B.4), valid at equilibrium, were used in 34 in an out-ofequilibrium context, for lattices of size $L=80$. In that work it had been noticed that the replicon is the only correlator that carries a significant signal.

Also in the present work we measured both signals, and we can confirm that the same phenomenology is observed in completely thermalised systems. In figure B1 we plot both the replicon susceptibility $\chi_{\mathrm{R}}$ and the longitudinal susceptibility $\chi_{\mathrm{L}}$, at $h=0.1,0.2$. The figure is qualitatively very similar to figure 13 of [34], where it is shown that $\chi_{\mathrm{R}}$ carries a significant signal, while $\chi_{\mathrm{L}}$ is very close to zero.

Appendix B.1. The effective anomalous dimension in the spin-glass phase

We can use the fact that $G_{\mathrm{R}}$ is dominant with respect to $G_{\mathrm{L}}$ to predict the value of the effective anomalous exponent $\eta_{\text {eff }}$ defined in section 9 in the deep spin-glass phase.

In fact, in a replica-symmetry breaking (RSB) situation the overlap $q$ has a finite support, so the overlap's variance $\sigma_{q}^{2}=E\left(q^{2}\right)-E(q)^{2}$ is of order one:

$$
\mathrm{RSB} \Rightarrow \sigma_{q}^{2} \sim 1
$$

Now, on general grounds (see for instance [72]) we can expect

$$
\left[E\left(q^{2}\right)-E(q)^{2}\right] \sim \overline{\left\langle q^{2}\right\rangle-\langle q\rangle^{2}}
$$

and remark that the r.h.s. is $\hat{\Gamma}_{2}(\mathbf{0}) / V$, the zero-moment Fourier transform of $\Gamma_{2}$ [defined in $(B .5)]$. We have then that in RSB conditions

$$
\Gamma_{2}(\mathbf{0}) \sim V \sigma_{q}^{2} \stackrel{\mathrm{RSB}}{\sim} V .
$$

$\Gamma_{2}$ can be related to the replicon and longitudinal susceptibilities through (B.5), that imply that $\Gamma_{2}(\mathbf{0})=\chi_{\mathrm{R}}+\frac{1}{2} \chi_{\mathrm{L}}$. Now, in the beginning of this section we found out empirically that the longitudinal susceptibility is subdominant with respect to the replicon channel (figure B1, so in the large-volume limit, in the presence of RSB, the replicon susceptibility scales like the volume:

$$
\mathrm{RSB} \Rightarrow \chi_{R} \sim V \text {. }
$$

Let us recall (29) and impose the just-found implication. We have then

$$
2^{D} \stackrel{\mathrm{RSB}}{=} \frac{\chi_{\mathrm{R}, 2 L}}{\chi_{\mathrm{R}, L}} \equiv 2^{2-\eta_{\mathrm{eff}}},
$$

therefore in the spin-glass phase we would have $\eta_{\mathrm{eff}}=-1$.

$\sharp$ In the effective field theory the longitudinal $\left(G_{\mathrm{L}}\right)$ and anomalous $\left(G_{\mathrm{A}}\right)$ propagators are degenerated. 


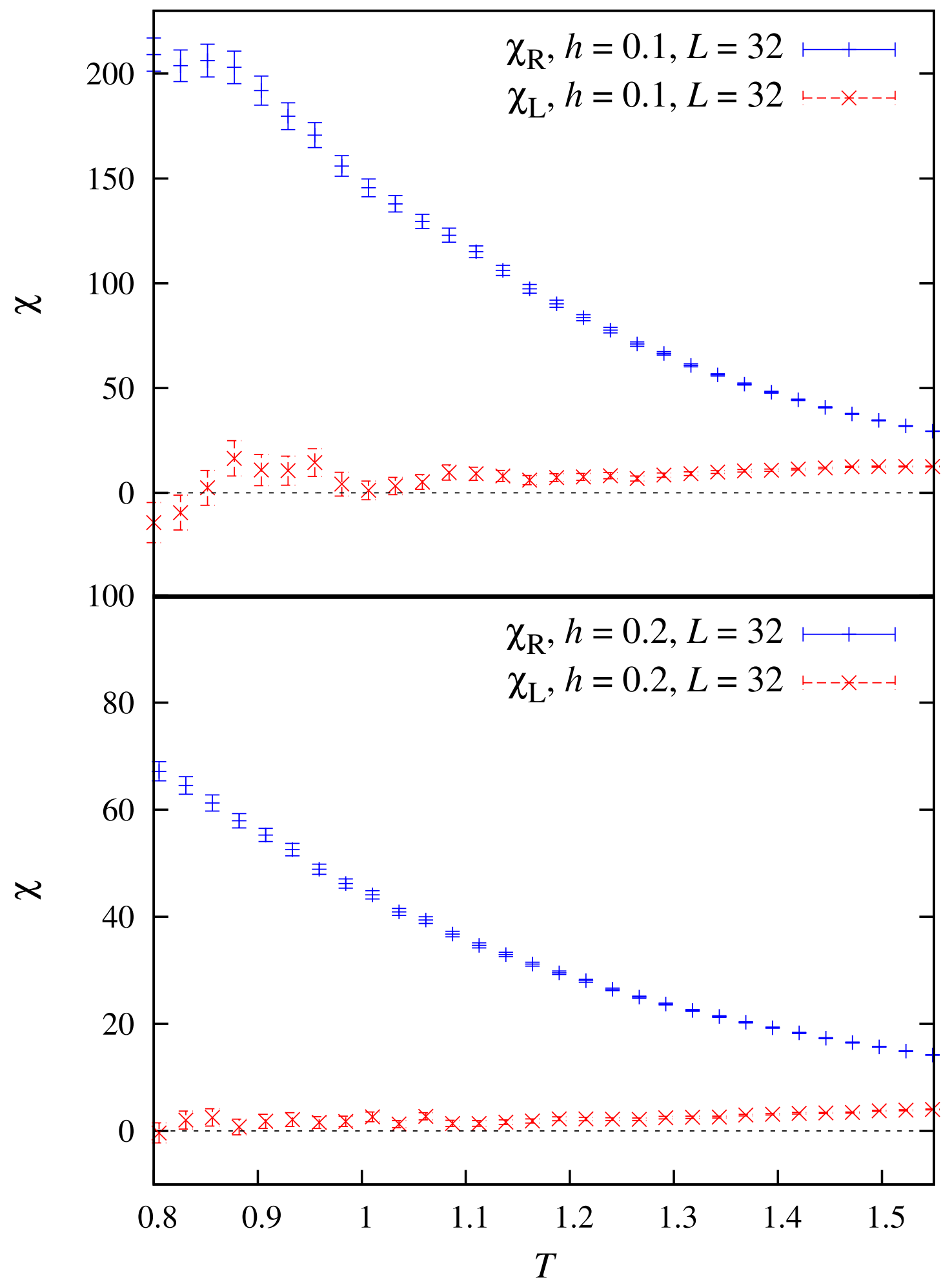

Figure B1. Replicon and longitudinal susceptibility as a function of $T$ in our equilibrium simulations, for the fields $h=0.1,0.2$ in our largest lattice sizes $(L=32)$. Just as in 34 the signal carried by the longitudinal propagator is much smaller than that of the replicon. 


\section{Appendix C. Technical details on the creation of quantiles}

To grant the reproducibility of our results, we give some details on how we proceeded in the labelling of the observables with the conditioning variate, and over the definition of the quantiles.

\section{Appendix C.1. Creating the $P(\hat{q})$}

As already explained in section 3 the analysis we conduct uses instantaneous realisations of the observables, instead of the average over the equilibrium regime. This is because computing $P(\hat{q})$ properly requires as many instances of the overlap as possible.

Operatively, we divide the second half of the simulation time-series in 16 blocks, and for the 4 replicas we save the final configuration of each block. This gives us $16^{4}$ configurations over which we can potentially compute overlaps for a single sample. For $N_{\mathrm{t}}$ times we pick 4 random numbers between 1 and 16 to create an instant measure. This way we increase our statistics of a factor $N_{\mathrm{t}}$, obtaining $\mathcal{N}_{\mathrm{m}}=N_{\text {samples }}(L, T, h) \times N_{\mathrm{t}}$ measures for each triplet $(L, T, h)$. We used $N_{\mathrm{t}}=1000$.

With the 4 replicas it is possible to compute 6 different overlaps $q_{i}(i=1, \ldots, 6)$, and one instance of most observables, for example the replicon susceptibility $\chi_{\mathrm{R}}$. Our ansatz is that $\chi_{\mathrm{R}}$ and the overlaps have some type of correlation, so we label $\chi_{\mathrm{R}}$ with some function of the overlaps $\hat{q}\left(q_{1}, \ldots, q_{6}\right)$, that we called conditioning variate.

The random variable $\hat{q}$ will have a probability distribution function $P(\hat{q})$ that we want to calculate numerically, in order to be able to work on the quantiles. Since our objective is not to individuate exactly the quantiles, but to compute observables related to a particular quantile, we coarse grain the range of definition of the $P(\hat{q})$. This is done by making a binning of the $P(\hat{q})$. This way, each conditioned expectation value of a generic observable, $E(\mathcal{O} \mid \hat{q})$, can be calculated over a reasonable amount of measurements, and we have exactly one conditioned expectation value for each bin of the $P(\hat{q})$. Integrals such as those in $(18)$ and $(19)$ are computed as sums over the histogram bins. Furthermore, the described histogramming procedure has the advantage that errors can be calculated in a very natural way with the jackknife method.

In order to have, as $L$ increases, both a growing number of bins, and of points per bin, we choose bins of width $\Delta \hat{q}=1 / \sqrt{a V}$. We add the restriction of having at least 150 bins, in order to be able to define the quantiles properly (we want to avoid the eventuality of two quantiles between the same two bins). We verified that there is no appreciable difference in the results between $a=1,2,4$. Larger $a$ implies a too large error, because the bins are too small, while with smaller $a$ the bins are too few. The results we show throughout this article have $a=2$.

To compute the conditional expectation values defined in section 6 we use the following estimators:

$$
E(\mathcal{O} \mid \hat{q}=c) \approx \frac{\frac{1}{\mathcal{N}_{\mathrm{m}}} \sum_{i}^{\mathcal{N}_{\mathrm{m}}} \mathcal{O}_{i} \mathcal{X}_{c}\left(\hat{q}_{i}\right)}{\frac{1}{\mathcal{N}_{\mathrm{m}}} \sum_{i}^{\mathcal{N}_{\mathrm{m}}} \mathcal{X}_{c}\left(\hat{q}_{i}\right)}
$$




$$
P(\hat{q}) \quad \approx \frac{1}{\mathcal{N}_{\mathrm{m}}} \sum_{i}^{\mathcal{N}_{\mathrm{m}}} \mathcal{X}_{c=\hat{q}}\left(\hat{q}_{i}\right),
$$

where with the symbol " $\approx$ " we stress that the quantity is an estimator that converges to the exact value only in the limit of an infinite number of measurements.

\section{Appendix C.2. Defining the quantiles}

As stated in section 7, the quantiles are the points that separate definite areas under $P(\hat{q})$. Therefore, the $i^{\text {th }}$ quantile $\tilde{q}_{i}$ is defined by means of the cumulative distribution $X(\hat{q})$ of $P(\hat{q})$, via the implicit relation

$$
X\left(\tilde{q}_{i}\right)=\int_{-1}^{\tilde{q}_{i}} \mathrm{~d} \hat{q} P(\hat{q})=\frac{i}{10} .
$$

Since this is a continuous relation, and our binning is discrete, it is most probable that the quantile fall between two neighbouring bins. To evaluate the observables right at the position of the quantile, we make linear interpolations between the two bins.

Let us call $i_{\text {bin }}^{-}\left(i_{\text {bin }}^{+}\right)$the bin just under (over) quantile $i$. Observable $\mathcal{O}_{i}$ at quantile $i$ will be a linear combination of the values it assumes at $i_{\mathrm{bin}}^{-}$and $i_{\mathrm{bin}}^{+}$:

$$
\mathcal{O}_{i}=p \mathcal{O}_{i_{\text {bin }}^{-}}+(1-p) \mathcal{O}_{i_{\text {bin }}^{+}}
$$

where the interpretation of the indices is straightforward, and $0 \leq p \leq 1$ is the interpolation weight

$$
p=\frac{X\left(\tilde{q}_{i}\right)-X\left(\hat{q}_{i_{\text {bin }}^{+}}\right)}{X\left(\hat{q}_{i_{\text {bin }}^{-}}\right)-X\left(\hat{q}_{i_{\text {bin }}^{+}}\right)} .
$$

\section{Appendix D. A caveat for the quantile description}

In the absence of an applied field, the overlap probability distribution function $P(q)$ is symmetric, with a single peak centred in $q=0$. In the presence of a field, instead, we expect the $P(q)$ to be strictly positive, at least in the thermodynamic limit. Similarly, we expect that the probability distribution function $P\left(q_{\mathrm{med}}\right)$ have only one peak at positive $q_{\text {med }}$ when a field is applied, and a peak in $q=0$ if $h=0$.

If the system sizes are too small, it may occur that the $h=0$ behavior bias the $P\left(q_{\text {med }}\right)$. This is what happens, for example, when $L=6, h=0.2$ and the temperature is sufficiently low: a second peak around $q_{\text {med }} \simeq 0$ develops upon lowering $T$ (figure D1, top). This second peak disappears when we increase the lattice size (figure D1, centre), and the $P\left(q_{\text {med }}\right)$ assumes only positive values when $L$ is large enough (figure D1, bottom). The lower the field, the easier it is to find multiple peaks, and the greater the system has to be to be able to neglect the $h=0$ behavior. For $h=0.05$, even lattices with $L=12$ show a double peak.

A second peak in $P\left(q_{\text {med }}\right)$ is a clear signal that we are observing and echo of $h=0$. When we make the quantile classification, and have a quantile on a peak, we are seeing 


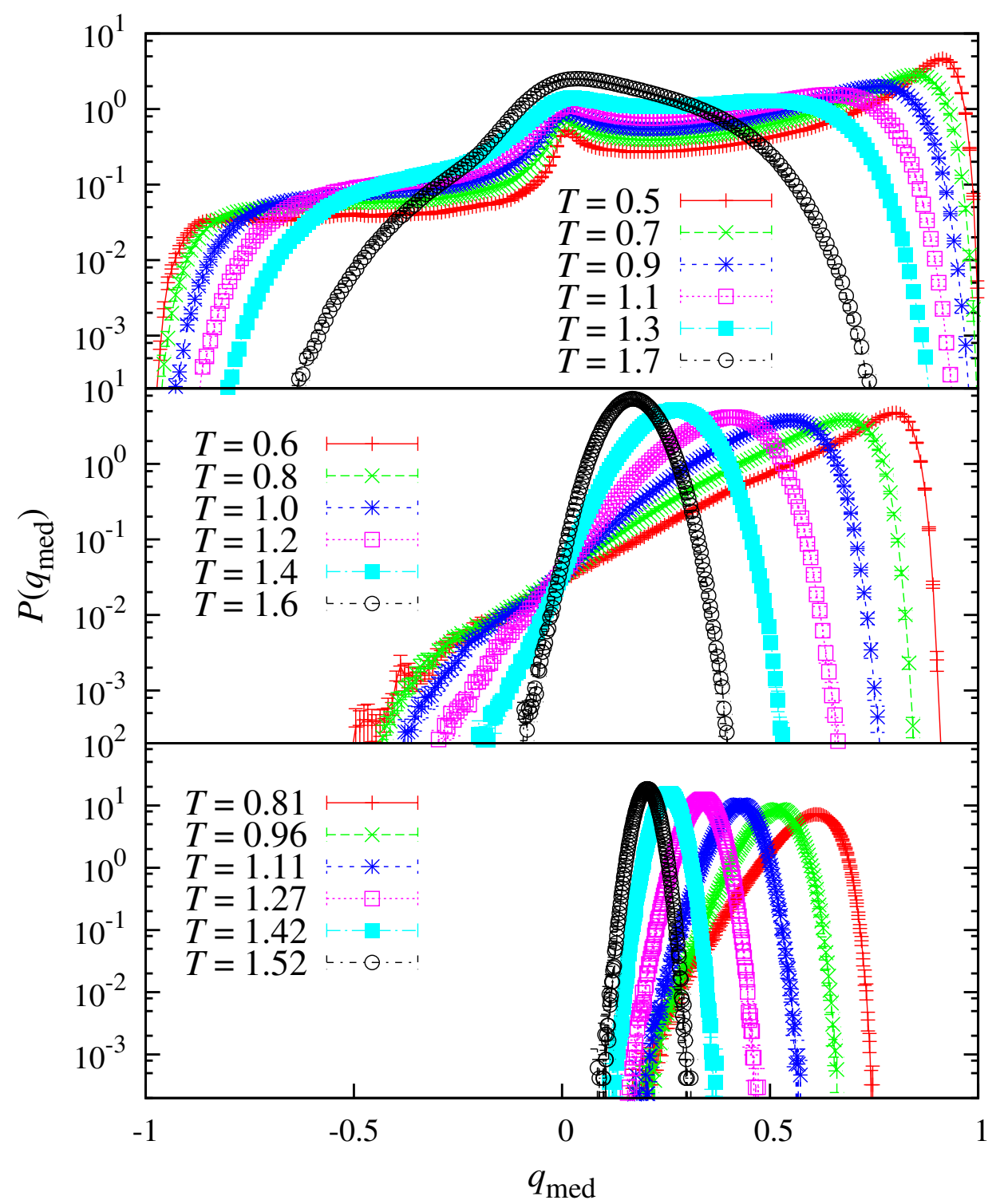

Figure D1. Median overlap probability distribution function $P\left(q_{\text {med }}\right)$ with $h=0.2$ for different temperatures (the ones from $L=32$ are an approximation to the second decimal digit). The top figure shows the case of $L=6$, where the lowest temperature curves display a second peak around $q_{\text {med }} \simeq 0$, which disappears when $T$ increases. For $L=16$, the $P\left(q_{\text {med }}\right)$ are single-peaked, but assume also negative values. In the bottom curve we have $L=32$, where the $P\left(q_{\text {med }}\right)$ are single-peaked and defined only on positive $q_{\text {med }}$, since we are closest to the asymptotic behavior.

only non-asymptotic data. Thus, quantile 1 for the smallest lattices gives us no relevant information.

If we plot any observable $\mathcal{O}$ related to quantile 1 versus the temperature, the information will be biased for low temperatures, and the bias will gradually disappear as 
we increase $T$. The result is that the curve $\mathcal{O}(T)$ will have a strange shape and will be of no use (see, e.g., the $h=0.05$ data in figure E1). This is why we did not include the $L=6$ points in figure 8 .

\section{Appendix E. Finding a privileged $q$}

Since all our simulations are in the paramagnetic phase the thermodynamic limit of the $P(q)$ is a delta function, so all the quantiles should tend to the a common $q=q_{\mathrm{EA}}$ in the $L \rightarrow \infty$ limit. We tried to perform these extrapolations at fixed (reasonably low) temperature, to see if we could look at the problem from such a privileged position. In figure E1 we see this type of extrapolation for $h=0.4$ and $h=0.05$, at temperatures $T=0.81$ and 1.109. The first is the lowest temperature we simulated in all our lattices, while the second is the zero-field critical temperature [51]. Since we are in the paramagnetic phase and we are plotting $\tilde{q}_{i}$ versus the inverse lattice size, the curves should cross at the intercept. This is indeed what appears to happen, but although in the case of $h=0.4$, the extrapolations were clean, for all the other simulated fields the finite-size effects were too strong and nonlinear to make solid extrapolations. We remark, yet, that once $L>8$ the $5^{\text {th }}$ quantile is the one with the least finite-size effects.

\section{Appendix F. Quantiles with 2-replica correlators}

To have well behaving (connected) correlators in the presence of a magnetic field we needed to use 4 replicas for each instance of them. As explained in sections 6 and 7 . since the overlap is a 2-replica observable, we had to choose a function of the 6 overlaps in order to have a one-to-one correspondence between conditioning variates and the correlators. The functions we tried out were the minimum, the maximum, the median and the average of the 6 overlaps.

Now, it is legitimate to ask oneself if the fluctuations we observed would also be visible having $q$ as conditioning variate. Although this is not possible with the replicon correlation function $G_{\mathrm{R}}$, we can renounce to have a connected correlation function, and study the fluctuations of the 2-replica point-to-plane correlator

$$
G_{2}^{\mathrm{nc}}(r)=\sum_{y, z} E\left(q_{(0,0,0)} q_{(r, y, z)}\right)
$$

which allows us to have $q$ as a conditioning variate. $G_{2}^{\mathrm{nc}}(r)$ is the total correlation between the origin, $(0,0,0)$, and the plane $x=r$. Of course, one could equivalently consider the planes $y=r$ or $z=r$. One can displace freely the origin, as well. We average over all these $3 \mathrm{~V}$ choices.

At this point, it is possible to compare with previous work that studied fluctuations with 2-replica correlators [47]. Furthermore, we can construct the pseudoconnected correlation function

$$
G_{2}^{\mathrm{c}}(r)=\frac{G_{2}^{\mathrm{nc}}(r)-G_{2}^{\mathrm{nc}}(L / 2)}{G_{2}^{\mathrm{nc}}(0)-G_{2}^{\mathrm{nc}}(L / 2)},
$$




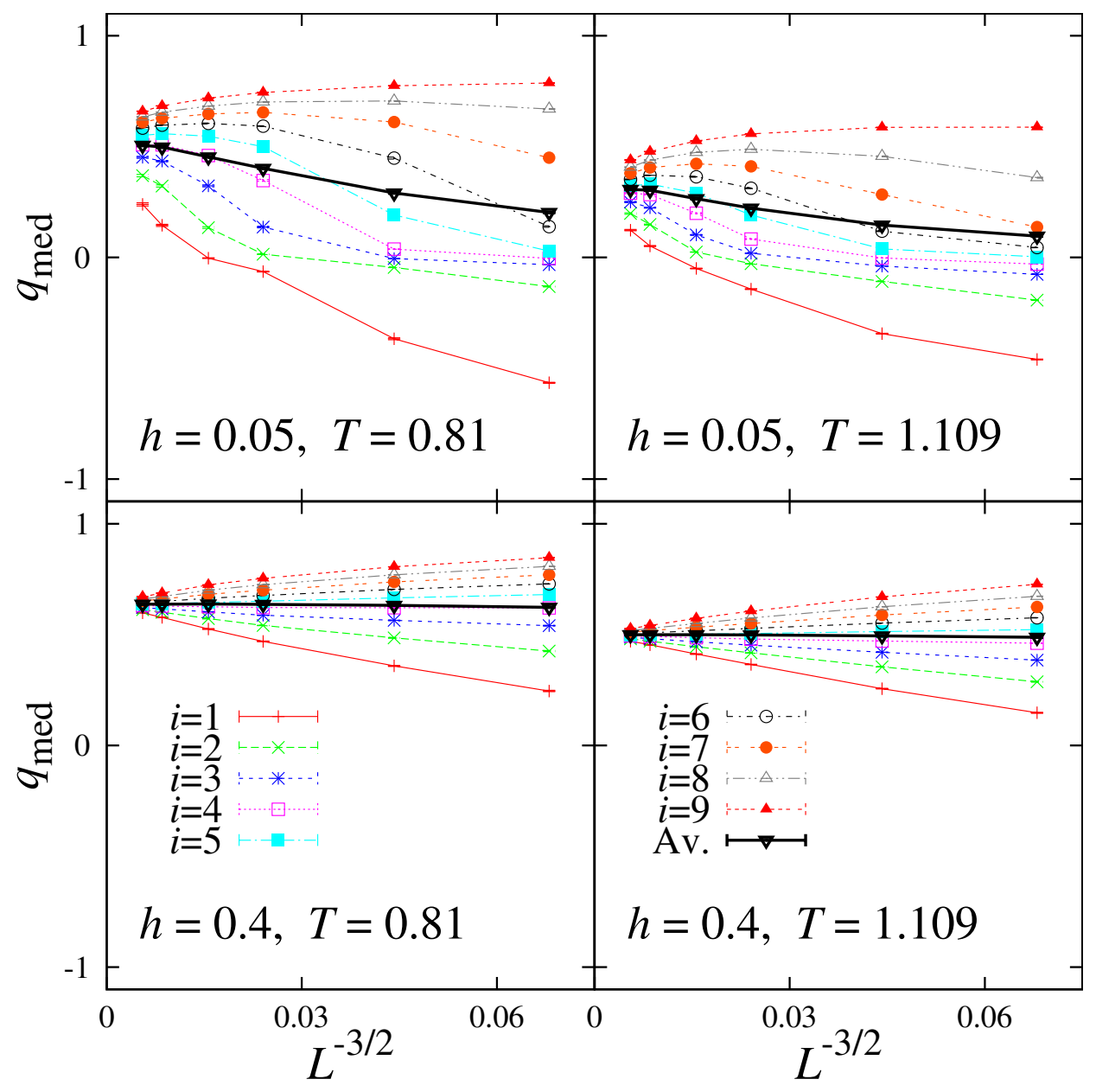

Figure E1. Extrapolations to infinite size of the quantile overlap $\tilde{q}_{i}$, for $T=0.81$ (left) and $T=1.109$ (right), and fields $h=0.05$ (top) and $h=0.4$ (bottom). We show quantiles $i=1, . .9$ (thin lines), and the average behavior (bold line). The $h=0.4$ extrapolations to infinite volume were clean $\left(\chi^{2} / \mathrm{DOF}<1\right.$ ), while for $h=0.05$ (and all the other fields we simulated), we encountered too strong and nonlinear finite-size effects to get reasonable extrapolations. We choose $1 / L^{D / 2}$ as scaling variable because in conditions of validity of the central limit theorem, the fluctuations should be of order $1 / \sqrt{V}$.

which forcedly is one for $r=0$, and goes to zero for $r=L / 2$. In figure F1 we show that the same dramatic fluctuations encountered with $G_{\mathrm{R}}$ (figure 3 ) are also present here.

The overall results, figure F2, are consistent with the picture we draw in the main part of the paper. On the one hand, the standard data average hides all signs of a phase transition. On the other hand, the fifth quantile displays signs of scale invariance.

\section{Appendix G. High-temperature extrapolation of the critical line}

While this paper was being completed, M. A. Moore and J. Yeo pointed out to 


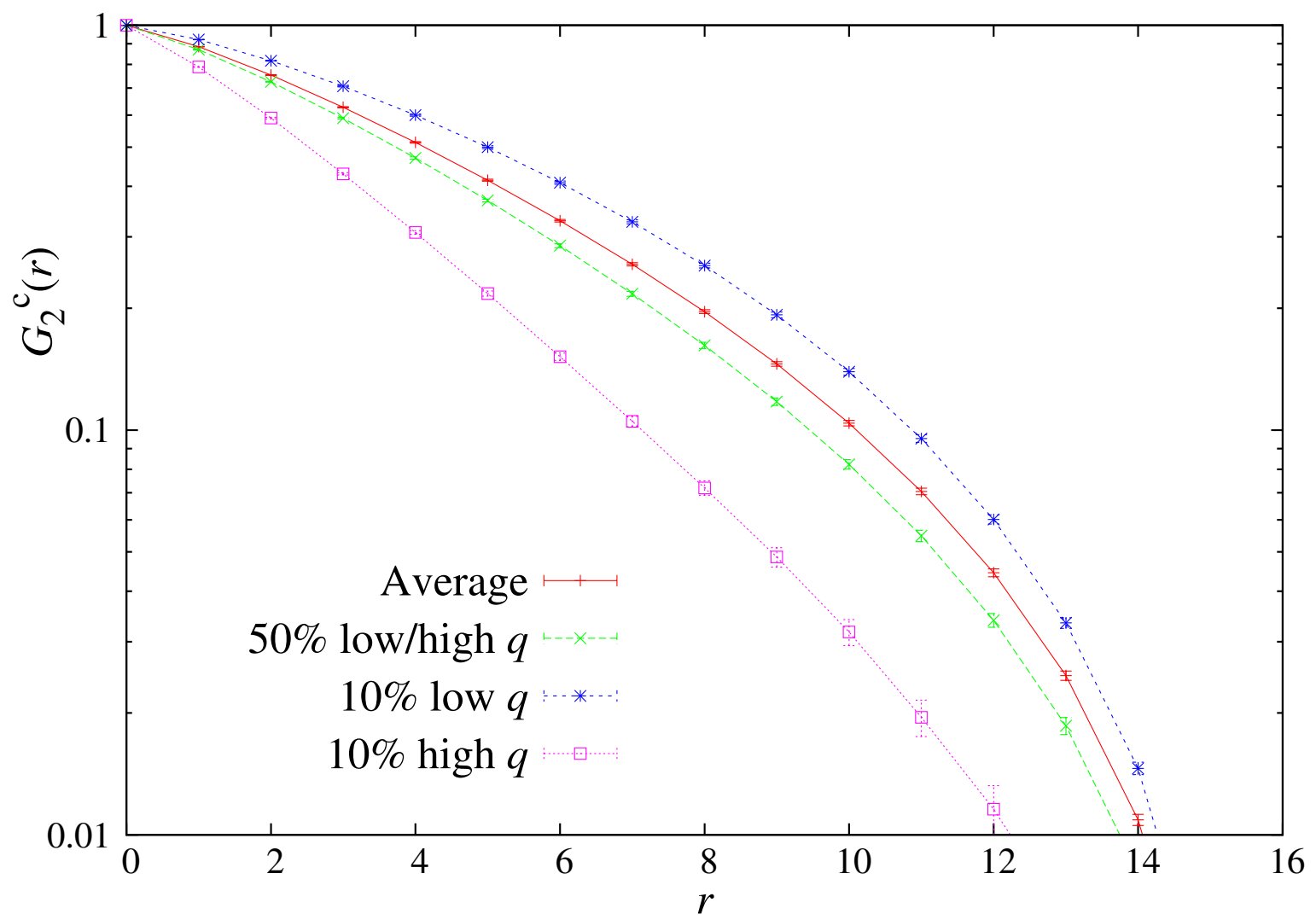

Figure F1. Same as figure 3, but for the 2-replica connected correlation function $G_{2}^{\mathrm{c}}(r)$ F.2. We show $L=32$ data from $h=0.2, T=0.805128$. Note that $G_{2}^{\mathrm{c}}(r)$ is bound to be 1 at $r=0$, and 0 at $r=L / 2$, so the fluctuations between different quantiles are even stronger than they may appear.
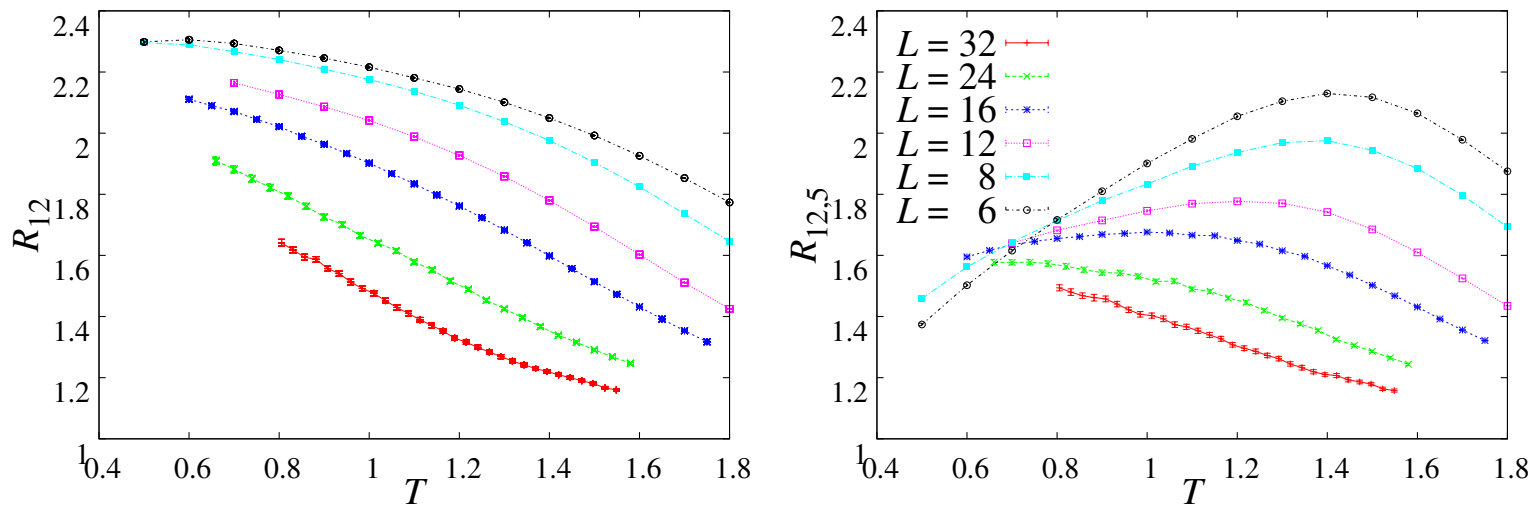

Figure F2. The $R_{12}$ cumulant computed from the two-replica correlation function (F.1) rather than from four replicas. The field is $h=0.2$. On the left side we show the average behavior, and on the right, the $5^{\text {th }}$ quantile, with the plain overlap $q(3)$ as conditioning variate.

us that it would be worthy to consider the Fisher-Sompolinsky scaling [73] for the correlation-length data from [34]. Since these data were obtained in a very large lattice 


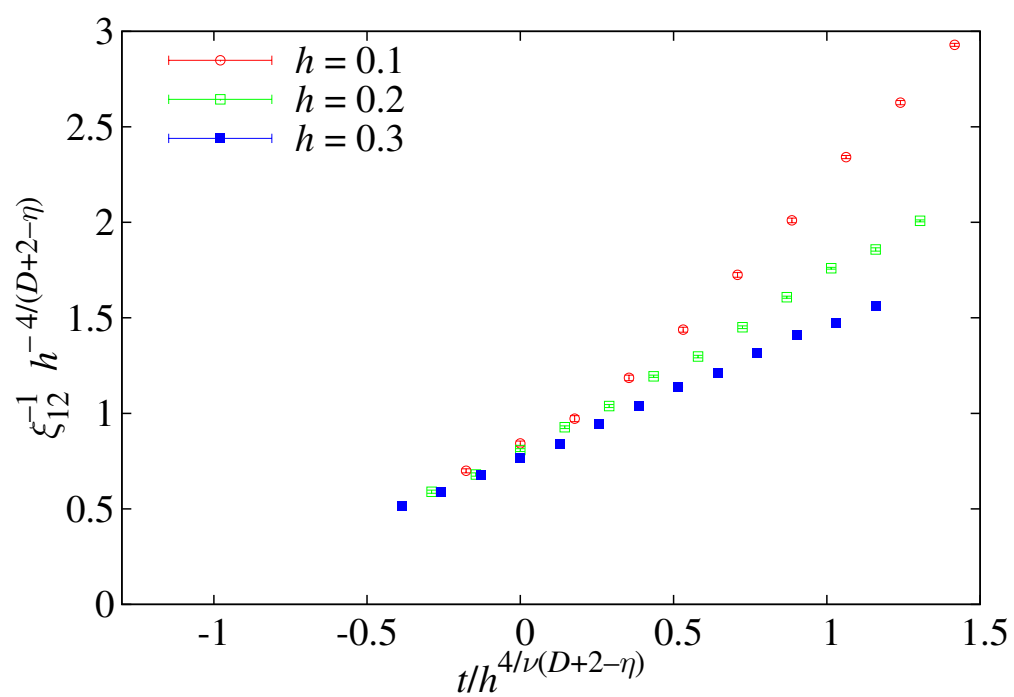

Figure G1. The $\xi_{12}$ estimate of the correlation length, from the thermodynamic-limit data in [34]. We display the data as suggested by the Fisher-Sompolinsky scaling (G.1). We plot $1 / \xi$, rather than $\xi$, in order to identify a possible critical point.

at comparatively high temperatures, the large fluctuations discussed in this paper should be much attenuated. Therefore, the results in [34] are to be regarded as representative of the thermodynamic limit in the paramagnetic phase.

The Fisher-Sompolinsky scaling suggests a possible location of the dAT line in rough agreement with our finite-size scaling analysis at lower temperatures. Let us see how it works.

A standard RG argument (see, e.g., [66]) tells us that, at least for small magnetic fields and close to the zero-field critical temperature, the correlation length should behave as

$$
h^{2 / y_{h}} \xi(t, h)=F\left(t / h^{2 y_{t} / y_{h}}\right),
$$

where $t$ is the $h=0$ reduced temperature, $t=\left(T-T_{\mathrm{c}}\right) / T_{\mathrm{c}}$, while the $h=0$ critical exponents are

$$
y_{h}=\frac{D+2-\eta}{2}, \quad y_{t}=\frac{1}{\nu} .
$$

We shall assess G.1] using the estimates of [51] $\dagger \dagger$

In figure G1 we plot $y=1 /\left[h^{2 / y_{h}} \xi(t, h)\right]$ as a function of the scaling variable $x=t / h^{2 y_{t} / y_{h}}$. At the (would-be) dAT line, $y$ should vanish at a critical $x_{c}$. In fact, $y$ should behave as $y \sim\left(x_{\mathrm{c}}-x\right)^{\nu_{h}}$ (i.e., $\nu_{h}$ is the correlation-length exponent in a field). Hence, at least for small $h$, one would have

$$
T_{\mathrm{c}}(h)=T_{\mathrm{c}}(h=0)\left[1+x_{\mathrm{c}} h^{2 y_{t} / y_{h}}\right]
$$

$\dagger \dagger$ The correlation length in [34 is not exactly the same computed here (technically, it is the so-called $\xi_{12}$ integral estimator [59]). Yet, the difference is immaterial as far as scaling properties are concerned. 
Now, the scaling in figure G1 is poor, but it improves upon decreasing $x$ (i.e., lowering the temperature). The estimation of $x_{\mathrm{c}}$ depends strongly on the value that one uses for exponent $\nu_{h}$. For instance, the rather improbable value $\nu_{h}=1$ suggests $x_{\mathrm{c}} \approx-1.2$. With a rather more plausible $\nu_{h}=\nu \approx 2.56$ one would guess $x_{\mathrm{c}} \approx-0.6$. This disparate range of $x_{c}$ would predict $T_{\mathrm{c}}(h=0.1) \approx 0.4-0.8, T_{\mathrm{c}}(h=0.2) \approx 0.3-0.7$, $T_{\mathrm{c}}(h=0.4) \approx 0.1-0.6$. Overall, these numbers are not in conflict with the finite-size scaling analysis we presented, but they are not of much help to locate of the dAT line.

\section{References}

[1] Mydosh J A 1993 Spin Glasses: an Experimental Introduction (London: Taylor and Francis)

[2] Edwards S F and Anderson P W 1975 J. Phys. F 5965

[3] Edwards S F and Anderson P W 1976 J. Phys. F 61927

[4] Mézard M, Parisi G and Virasoro M 1987 Spin-Glass Theory and Beyond (Singapore: World Scientific)

[5] de Almeida J R L and Thouless D J 1978 J. Phys. A 11983

[6] Marinari E, Parisi G, Ricci-Tersenghi F, Ruiz-Lorenzo J J and Zuliani F 2000 J. Stat. Phys. 98 973 (Preprint arXiv: cond-mat/9906076)

[7] Parisi G 1979 Phys. Rev. Lett. 431754

[8] Parisi G 1980 J. Phys. A: Math. Gen. 131101

[9] Parisi G 1983 Phys. Rev. Lett. 501946

[10] Parisi G and Temesvári T 2012 Nucl. Phys. B 858293 (Preprint arXiv:1111.3313)

[11] McMillan W L 1984 J. Phys. C: Solid State Phys. 173179

[12] Bray A J and Moore M A 1987 Scaling theory of the ordered phase of spin glasses Heidelberg Colloquium on Glassy Dynamics (Lecture Notes in Physics no 275) ed van Hemmen J L and Morgenstern I (Berlin: Springer)

[13] Fisher D S and Huse D A 1986 Phys. Rev. Lett. 561601

[14] Fisher D S and Huse D A 1988 Phys. Rev. B 38386

[15] Moore M A and Bray A J 2011 Phys. Rev. B 83224408 (Preprint arXiv:1102.1675)

[16] Yeo J and Moore M A 2012 Phys. Rev. E 86052501 (Preprint arXiv:1208.3044)

[17] Jönsson P E, Takayama H, Katori H A and Ito A 2005 Phys. Rev. B 71 180412(R) (Preprint arXiv: cond-mat/0411291)

[18] Petit D, Fruchter L and Campbell I A 1999 Phys. Rev. Lett 835130 (Preprint arXiv:cond-mat/ 9910353)

[19] Petit D, Fruchter L and Campbell I A 2002 Phys. Rev. Lett 88207206 (Preprint arXiv: cond-mat/011112)

[20] Tabata Y, Matsuda K, Kanada S, Yamazaki T, Waki T, Nakamura H, Sato K and Kindo K 2010 Journal of Physical Society of Japan 79123704 (Preprint arXiv:1009.6115)

[21] Bray A J and Moore M A 1980 J. Phys. C: Solid St. Phys. C 13419

[22] Temesvári T and De Dominicis C 2002 Phys. Rev. Lett. 89097204 (Preprint arXiv:cond-mat/ 0207512)

[23] Temesvári T 2008 Phys. Rev. B 78220401

[24] Palassini M and Caracciolo S 1999 Phys. Rev. Lett. 82 5128-5131 (Preprint arXiv:cond-mat/ 9904246)

[25] Ballesteros H G, Cruz A, Fernandez L A, Martin-Mayor V, Pech J, Ruiz-Lorenzo J J, Tarancon A, Tellez P, Ullod C L and Ungil C 2000 Phys. Rev. B 62 14237-14245 (Preprint arXiv: cond-mat/0006211)

[26] Gunnarsson K, Svedlindh P, Nordblad P, Lundgren L, Aruga H and Ito A 1991 Phys. Rev. B 43 8199-8203 
[27] Young A P and Katzgraber H G 2004 Phys. Rev. Lett. 93207203 (Preprint arXiv:cond-mat/ 0407031)

[28] Jörg T, Katzgraber H G and Krzakala F 2008 Phys. Rev. Lett. 100197202 (Preprint arXiv: 0712.2009)

[29] Baños R A, Cruz A, Fernandez L A, Gil-Narvion J M, Gordillo-Guerrero A, Guidetti M, Iniguez D, Maiorano A, Marinari E, Martin-Mayor V, Monforte-Garcia J, Muñoz Sudupe A, Navarro D, Parisi G, Perez-Gaviro S, Ruiz-Lorenzo J J, Schifano S F, Seoane B, Tarancon A, Tellez P, Tripiccione R and Yllanes D 2012 Proc. Natl. Acad. Sci. USA 1096452

[30] Kotliar G, Anderson P W and Stein D L 1983 Phys. Rev. B 27602

[31] Leuzzi L, Parisi G, Ricci-Tersenghi F and Ruiz-Lorenzo J J 2008 Phys. Rev. Lett. 101107203

[32] Larson D, Katzgraber H G, Moore M A and Young A P 2013 Phys. Rev. B 87024414 (Preprint arXiv:1211.7297)

[33] Leuzzi L and Parisi G 2013 Phys. Rev. B 88224204 (Preprint arXiv:1303.6333)

[34] Baity-Jesi M, Baños R A, Cruz A, Fernandez L A, Gil-Narvion J M, Gordillo-Guerrero A, Iniguez D, Maiorano A, F M, Marinari E, Martin-Mayor V, Monforte-Garcia J, Muñoz Sudupe A, Navarro D, Parisi G, Perez-Gaviro S, Pivanti M, Ricci-Tersenghi F, Ruiz-Lorenzo J J, Schifano S F, Seoane B, Tarancon A, Tripiccione R and Yllanes D 2014 Phys. Rev. E (in press) (Preprint arXiv:1307.4998)

[35] Belletti F, Guidetti M, Maiorano A, Mantovani F, Schifano S F, Tripiccione R, Cotallo M, PerezGaviro S, Sciretti D, Velasco J L, Cruz A, Navarro D, Tarancon A, Fernandez L A, Martin-Mayor V, Muñoz-Sudupe A, Yllanes D, Gordillo-Guerrero A, Ruiz-Lorenzo J J, Marinari E, Parisi G, Rossi M and Zanier G (Janus Collaboration) 2009 Computing in Science and Engineering 11 48

[36] Alvarez Baños R, Cruz A, Fernandez L A, Gil-Narvion J M, Gordillo-Guerrero A, Guidetti M, Maiorano A, Mantovani F, Marinari E, Martin-Mayor V, Monforte-Garcia J, Muñoz Sudupe A, Navarro D, Parisi G, Perez-Gaviro S, Ruiz-Lorenzo J J, Schifano S F, Seoane B, Tarancon A, Tripiccione R and Yllanes D (Janus Collaboration) 2010 J. Stat. Mech. 2010 P06026 (Preprint arXiv:1003.2569)

[37] Baños R A, Cruz A, Fernandez L A, Gil-Narvion J M, Gordillo-Guerrero A, Guidetti M, Iñiguez D, Maiorano A, Mantovani F, Marinari E, Martin-Mayor V, Monforte-Garcia J, Muñoz Sudupe A, Navarro D, Parisi G, Perez-Gaviro S, Ricci-Tersenghi F, Ruiz-Lorenzo J J, Schifano S F, Seoane B, Tarancón A, Tripiccione R and Yllanes D 2011 Phys. Rev. B 84(17) 174209 (Preprint arXiv:1107.5772) URL http://link.aps.org/doi/10.1103/PhysRevB.84.174209

[38] Baity-Jesi M, Baños R A, Cruz A, Fernandez L A, Gil-Narvion J M, Gordillo-Guerrero A, Guidetti M, Iniguez D, Maiorano A, Mantovani F, Marinari E, Martin-Mayor V, Monforte-Garcia J, Munoz Sudupe A, Navarro D, Parisi G, Pivanti M, Perez-Gaviro S, Ricci-Tersenghi F, RuizLorenzo J J, Schifano S F, Seoane B, Tarancon A, Tellez P, Tripiccione R and Yllanes D 2012 Eur. Phys. J. Special Topics 21033 (Preprint arXiv:1204.4134)

[39] Debenedetti P G 1997 Metastable Liquids (Princeton: Princeton University Press)

[40] Debenedetti P G and Stillinger F H 2001 Nature 410 259-267

[41] Cavagna A 2009 Physics Reports 476 51-124 (Preprint arXiv:0903.4264)

[42] Castellani T and Cavagna A 2005 J. Stat. Mech. 2005 P05012

[43] Kirkpatrick T R and Thirumalai D 1987 Phys. Rev. B 365388

[44] Kirkpatrick T R, Thirumalai D and Wolynes P G 1989 Phys. Rev. A 401045

[45] Moore M A and Drossel B 2002 Phys. Rev. Lett. 89217202 (Preprint arXiv:cond-mat/0201107)

[46] Fullerton C J and Moore M A 2013 (Preprint arXiv:1304.4420)

[47] Parisi G and Ricci-Tersenghi F 2012 Phil. Mag. B 92341 (Preprint arXiv:1108.0759v1)

[48] Nightingale M P 1975 Physica A 83561

[49] Binder K 1982 Phys. Rev. A 251699

[50] Ballesteros H G, Fernandez L A, Martin-Mayor V, Muñoz Sudupe A, Parisi G and Ruiz-Lorenzo J J 1998 Nucl. Phys. B 512681 
[51] Baity-Jesi M, Baños R A, Cruz A, Fernandez L A, Gil-Narvion J M, Gordillo-Guerrero A, Iniguez D, Maiorano A, Mantovani F, Marinari E, Martin-Mayor V, Monforte-Garcia J, Muñoz Sudupe A, Navarro D, Parisi G, Perez-Gaviro S, Pivanti M, Ricci-Tersenghi F, Ruiz-Lorenzo J J, Schifano S F, Seoane B, Tarancon A, Tripiccione R and Yllanes D (Janus Collaboration) 2013 Phys. Rev. B 88224416 (Preprint arXiv:1310.2910)

[52] Baity-Jesi M, Fernandez L A, Martin-Mayor V and Sanz J M 2014 Phys. Rev. B 89(1) 014202 (Preprint arXiv:1309.1599)

[53] Lee L W and Young A P 2003 Phys. Rev. Lett. 90227203

[54] Jörg T 2006 Phys. Rev. B 73224431

[55] Fernandez L A, Martin-Mayor V and Yllanes D 2009 Nucl. Phys. B 807 424-454

[56] Campos I, Cotallo-Aban M, Martin-Mayor V, Perez-Gaviro S and Tarancon A 2006 Phys. Rev. Lett. 97217204

[57] Baños R A, Fernandez L A, Martin-Mayor V and Young A P 2012 Phys. Rev. B 86134416 (Preprint arXiv:1207.7014)

[58] Fernandez L A, Martin-Mayor V, Parisi G and Seoane B 2013 EPL 10367003 (Preprint arXiv:1307.2361)

[59] Belletti F, Cruz A, Fernandez L A, Gordillo-Guerrero A, Guidetti M, Maiorano A, Mantovani F, Marinari E, Martin-Mayor V, Monforte J, Muñoz Sudupe A, Navarro D, Parisi G, Perez-Gaviro S, Ruiz-Lorenzo J J, Schifano S F, Sciretti D, Tarancon A, Tripiccione R and Yllanes D (Janus Collaboration) 2009 J. Stat. Phys. 1351121 (Preprint arXiv:0811.2864)

[60] Hukushima K and Nemoto K 1996 J. Phys. Soc. Japan 651604 (Preprint arXiv:cond-mat/ 9512035)

[61] Marinari E 1998 Optimized Monte Carlo methods Advances in Computer Simulation ed Kerstész $\mathrm{J}$ and Kondor I (Springer-Berlag)

[62] Newman M E J and Barkema G T 1999 Monte Carlo Methods in Statistical Physics (Oxford: Clarendon Press)

[63] Fernandez L A, Martin-Mayor V, Perez-Gaviro S, Tarancon A and Young A P 2009 Phys. Rev. B 80024422

[64] Yllanes D 2011 Rugged Free-Energy Landscapes in Disordered Spin Systems (Ph.D. thesis, UCM) (Preprint arXiv:1111.0266)

[65] de Dominicis C and Giardina I 2006 Random Fields and Spin Glasses (Cambridge, England: Cambridge University Press)

[66] Amit D J and Martin-Mayor V 2005 Field Theory, the Renormalization Group and Critical Phenomena 3rd ed (Singapore: World Scientific) URL http://www.worldscientific.com/ worldscibooks/10.1142/5715

[67] Ballesteros H G, Fernandez L A, Martin-Mayor V and Muñoz Sudupe A 1996 Phys. Lett. B 378 207 (Preprint arXiv:hep-lat/9511003)

[68] Fernandez L A and Martin-Mayor V 2009 Phys. Rev. E 79051109

[69] Alvarez Baños R, Cruz A, Fernandez L A, Gil-Narvion J M, Gordillo-Guerrero A, Guidetti M, Maiorano A, Mantovani F, Marinari E, Martin-Mayor V, Monforte-Garcia J, Muñoz Sudupe A, Navarro D, Parisi G, Perez-Gaviro S, Ruiz-Lorenzo J J, Schifano S F, Seoane B, Tarancon A, Tripiccione R and Yllanes D (Janus Collaboration) 2010 Phys. Rev. Lett. 105177202 (Preprint arXiv:1003.2943)

[70] Hyndman R and Fan Y 1996 American Statistician 50(4) 361

[71] de Dominicis C, Kondor I and Temesvári T 1998 Beyond the Sherrington-Kirkpatrick model Spin Glasses and Random Fields ed Young A P (Singapore: World Scientific) (Preprint arXiv: cond-mat/9705215)

[72] Fisher K and Hertz J 1991 Spin Glasses (Cambridge England: Cambridge University Press)

[73] Fisher D S and Sompolinsky H 1985 Phys. Rev. Lett. 541063 